\title{
A Novel Many-objective Evolutionary Algorithm Based on Transfer Matrix with Kriging model
}

Ma, L., Wang, R., Chen, S., Wang, X., Cheng, C., Lin, Z., \& Shi, Y. (Accepted/In press). A Novel Many-objective Evolutionary Algorithm Based on Transfer Matrix with Kriging model. Information Sciences.

Link to publication record in Ulster University Research Portal

\section{Published in:}

Information Sciences

Publication Status:

Accepted/In press: 11/01/2019

\section{Document Version}

Author Accepted version

\section{General rights}

Copyright for the publications made accessible via Ulster University's Research Portal is retained by the author(s) and / or other copyright owners and it is a condition of accessing these publications that users recognise and abide by the legal requirements associated with these rights.

\section{Take down policy}

The Research Portal is Ulster University's institutional repository that provides access to Ulster's research outputs. Every effort has been made to ensure that content in the Research Portal does not infringe any person's rights, or applicable UK laws. If you discover content in the Research Portal that you believe breaches copyright or violates any law, please contact pure-support@ulster.ac.uk. 


\title{
A Novel Many-objective Evolutionary Algorithm Based on Transfer Matrix with Kriging model
}

\author{
Lianbo Ma a , Rui Wang a , Shengminjie Chen ${ }^{\mathrm{b}}$, Xingwei Wang ${ }^{\mathrm{a}}, \mathrm{Chi}_{\text {Cheng }}^{\mathrm{c}}$, Zhiwei Lin ${ }^{\mathrm{d}}$, \\ Yuhui Shi \\ ${ }^{a}$ College of Software, Northeastern University, Shenyang, China \\ ${ }^{b}$ Faculty of Science, Kunming University of Science and Technology, Kunming, China \\ ${ }^{\mathrm{c}}$ School of Computer Science, Shaanxi Normal University, Xi' an, China \\ ${ }^{\mathrm{d}}$ School of Computing, Ulster University, United Kingdom \\ ${ }^{\mathrm{e}}$ Southern University of Science and Technology, Shenzhen, China
}

\begin{abstract}
Due to the curse of dimensionality caused by the increasing number of objectives, it is very challenging to tackle many-objective optimization problems (MaOPs). Aiming to alleviate the loss of selection pressure in the fitness evaluation for MaOPs, this paper proposes a novel evolutionary optimization framework, called Tk-MaOEA, based on transfer learning assisted by Kriging model. In this approach, in order to achieve global space optimization, transfer learning is used as a map tool to reduce the objective space, i.e., devising transfer matrix to simplify the optimization process. For the objective optimization, the Kriging model is appropriately incorporated in order to further reduce computation cost. Accordingly, any EA-based paradigm or search strategy can be integrated into this framework. Fast non-dominated sorting and farthest-candidate selection (FCS) methods are used to guarantee the diversity of non-dominated solutions. Comprehensive evaluations on a set of benchmark functions have been conducted to show that the proposed Tk-MaOEA is efficietive for solving complex MaOPs.
\end{abstract}

Key Words: Evolutionary algorithm, Many-objective optimization, Transfer matrix, Kring model.

\section{Introduction}

Multi-objective optimization problems (MOPs) occur in many real-world applications, in which multiple conflicting objectives need to be solved in order to find a set of optimal [1,2]. Accordingly, the solutions to these MOPs, referred as Pareto-optimal solutions (PS), denote a possible reasonable trade-off between all involved objectives. And the image of PS in the objective space is known as Pareto front (PF) [3, 4]. When MOPs have more than three objectives, they are called as many-objective optimization problems (MaOPs) [5-7]. As an effective optimization paradigm for MOPs, the multi-objective evolutionary algorithms (MOEAs) have been widely developed, being endowed with a powerful search ability to approximate the PF. However, most MOEAs, 
especially inevitably suffer from severe degradation in performance on MaOPs [5-9]. This is caused by the called curse of dimensionality w.r.t the difficulty of optimizing large number of objectives.

Experimental results $[8,9]$ have shown that traditional Pareto-dominance-based approaches, e.g., NSGA-II [10] and SPEA2 [11], encounter several serious difficulties when dealing with MaOPs as following.

First, compared with 2- or 3-objective MOPs, the high-dimensional MaOPs would render the Pareto optimality, which is unable to provide enough selection pressure to evolve the solutions towards the true PF. As the number of objectives increases, most of the obtained solutions become non-dominated to each other very quickly, resulting in the loss of selection pressure to drive the solutions to approximate the PF, which have been reported well in the literatures $[12,13]$. When the number of objectives rises over five, the proportion of non-dominated solutions in the population will reach more than $90 \%$ [14]. Thus, it is difficult to differentiate preferred solutions from innumerable non-dominated solutions obtained during the search process. Moreover, the many-objective optimization inevitably encounters the inability of exploring both convergence and diversity for the approximation of the true PF.

Second, the extensive search in a high-dimensional space would seriously undermine the efficiency of algorithmic operators, such as mating selection and variation [15]. As confirmed in [16, 17], in a variation process, the new offspring produced by two nearly converged solutions, which are required to approach along its original direction, would contrarily move far away the true PF. This causes the failure of the final population to converge to the PF, despite spreading all over the objective space. As a result, the EAs for MaOPs (also called MaOEAs) can only explore a limited region in a large search space, whereas being trapped in local segments of the PF due to the invalid evolutionary operators.

In addition, due to the large search space, the diversity based selection criterion would be harmful to facilitate the convergence of the obtained solutions, when it is activated in the selection process of non-dominated solutions. For example, experimental results in [13] show that the diversity maintenance mechanism in NSGA-II plays a negative role in the convergence performance on the 5-, and 10-objective DTLZ2 instances.

There are also some other problems, such as visualization of multi-dimensional objectives, high computation expense, and determination of an appropriate population size. Even if the PF is attainable, there are no effective methods to visualize the front. A large number of solutions in the high-dimensional objective space need to be selected and measured as the representations of PF, which is of computation cost.

In order to overcome the above difficulties, the dimensionality reduction scheme is naturally considered, to reduce the number of objectives while trying to maintain the information of the objectives as much as possible. 
For example, an effective approach, which uses the principle component analysis approach, has been proposed to determine the correlation between lower dimensions of each objective [18]. This approach relies on iterative progresses from the interior of the objective space towards the PF. A preset approximated front of non-dominated solutions is used to determine the redundant objectives [19]. However, in many real-world conditions, the problem's objectives sometimes cannot be reduced only according to the order of importance. This causes the ineffectiveness of above methods. Furthermore, even if a relatively small number of objectives can be reduced, it is not helpful to tackle the problem effectively in some specific cases.

This paper presents a new transfer learning method with Kriging model based MaOEA (Tk-MaOEA) without any reference vectors or points in advance, in order to alleviate effect of the curse of dimensionality in MaOPs. One of our main ideas is to reduce the complexity of large search space by using multi-dimensional compression based on transfer learning. At the global space optimization level, a new transfer learning approach is proposed to reduce the number of objectives, while the property of the objectives in the high-dimensional search space is still kept during the transferring process. In the proposed approach, the redundant dimensions are compressed using a transfer matrix with Gram-Schmidt orthogonalization. At the objective optimization level, Kriging models are utilized to reduce the number of expensive evaluations by approximating each objective value. By using these mechanisms, we can follow the idea of improving the effectiveness of Pareto optimality and overcoming the difficulty of the extremely large search space.

In Tk-MaOEA, the primary principle is to use transfer matrix for dimensionality reduction to enforce the population evolution to be limited in a low-dimensional search space. As a result, the simplified optimization in the small objective space not only guarantees the effectiveness of conventional evolutionary operators, but also facilitates improving the performance of Pareto optimality. Afterward, when optimizing the simplified low-dimensional MaOP, the Kriging model is constructed for each objective to enhance the objective optimization, by using the Latin hypercube sampling (LHS) method. In this proposed design, the transfer learning and Kriging model with FCS strategy perform distinctly, yet complementarily. Transfer learning offers the convergence power, while the FCS assisted by Kriging model enhances the primary diversity power.

Generally, the conventional work only focuses on the monotonous combination of surrogate models into conventional EA algorithms. In contrary, our design focuses the importance of the combinational contribution of the Kriging and transfer learning to the optimization goal. Tk-MaOEA utilizes and maximizes the benefits of Kriging model to assist the dimensionality reduction scheme for complex many-objective optimization. Our contributions mainly include:

1) At the global space optimization level, a new transfer learning approach is developed to reduce a large 
number of objectives while the original property of the problem is kept well. The proposed approach uses a specific transfer matrix to compress the search space, which is simple yet effective to handle with the curse of dimensionality in MaOPs.

2) At the objective optimization level, the Kriging model is devised for each objective to further reduce computational cost. This Bayesian based surrogate model is to measure not only the objective value itself but also stochastic error of the approximation, which is essentially conductive to improve the accuracy of the optimization.

3) The multi-scale normalization approach is employed so as to avoid the distortion caused by conventional normalization in the high-dimensional objective space. This is a significant operation for the MaOEA to keep unchanged spatial distribution when the original population are normalized.

4) The FCS approach is incorporated instead of the traditional crowding distance method in the environmental selection. This approach is more effective to select a set of representative non-dominated solutions in a single run.

The remainder of this paper is organized as follows. Section 2 elucidates related works. In Section 3 the proposed algorithm is given in detail. In Section 4, the experiment is conducted on a serial of well-defined test functions. Finally, Section 5 outlines the conclusions.

\section{Related works}

\subsection{Many-objective optimization}

An MOP with only box constraints is defined as follows:

$$
\begin{aligned}
& \underset{x}{\operatorname{Minimize} \quad F(x)=\left(f_{1}(x), \ldots, f_{m}(x)\right)} \\
& \text { s.t. } \quad\left\{\begin{array}{l}
g_{i}(x) \geq 0, \quad i=1,2, \cdots, k \\
h_{j}(x)=0, \quad j=1,2, \cdots, q
\end{array}\right.
\end{aligned}
$$

where $x=\left(x_{1}, \ldots, x_{n}\right)$ is $n$-dimensional decision vector from the decision space $R^{n} ; F: R^{n} \rightarrow R^{m}$ is a mapping function from $R^{n}$ to an objective space $R^{m}$, involving $m$ objectives; $k$ and $q$ are the number of inequality and equality constraints, respectively. If $m>3$, the problem is also referred as a $\mathrm{MaOP}$

1) Given two solutions $X_{1}, X_{2} \in R^{n}, X_{1}$ dominates $X_{2}$, i.e., $X_{1} \succ X_{2}$, if $\forall \mathrm{i} \in\{1,2, \ldots, m\}, f_{i}\left(X_{1}\right) \leq f_{i}\left(X_{2}\right)$ and $\exists \mathrm{i} \in\{1,2, \ldots, m\}, f_{i}\left(X_{1}\right)<f_{i}\left(X_{2}\right)$.

2) Any solution $x \in R^{n}$ can be referred as a Pareto-optimal solution or non-dominated solution if no other feasible solutions dominate $x$ in $R^{n}$.

3) The set of all Pareto-optimal solutions in the objective space is said to be the Pareto set (PS), and the plotted non-dominated solutions or PS is called the Pareto front $(P F)$, i.e., $P F:=\left\{\left(f_{1}(x), \ldots, f_{k}(x)\right) \mid x \in P S\right\}$.

To tackle these MaOPs, many MaOEAs have been developed deliberately, including the following classes. 
First, decomposition-based algorithms divide a complex MaOP into a set of scalar sub-problems and solve them in a cooperative manner. For example, MOEA/D [20] uses the weighted sum or Chebyshev method to select individuals for next generation, while the neighborhood of subproblems are incorporated. Then, several variants have been proposed [17, 21, 22]. Reference [17] develops a new double-level archive mechanism based on the framework of MOEA/D to maintain both convergence and diversity of solutions, and reference [11] proposes an improved MOEA/D variant called MOEA/D-EGO to reduce the computation cost.

The second approach is based on the idea of quality indicators. These indicators can be directly used as the fitness assignment to guide the evolutionary process. IBEA [23] has exhibited a prominent ability of converging to PF at a high pace. However, the diversity of population is not maintained appropriately [23]. Accordingly, a novel indicator $S$ is used to improve the convergence and diversity simultaneously [24]. Likewise, in [25] an effective indicator $R_{2}$ is proposed in MOMBI. Another interesting approach, the hypervolume (HV), can measure both the convergence and diversity, but it consumes much computation cost [26].

The third one is the relaxed dominance based approach. Those algorithms strike to alleviate the inefficiency of Pareto dominance via enhancing the selection pressure, such as Pareto $\varepsilon$-dominance [27], Pareto $\alpha$-dominance [28] and controlling Pareto dominance area [29]. It has been validated experimentally that these approaches are more effective than traditional Pareto dominance. Furthermore, for the augment of selection pressure, several new strategies are developed to make a solution be dominated by others more probably. The prominent examples include fuzzy-Pareto dominance [30], L-optimality [31], and ranking method [32]. Among those, one excellent approach GrEA [33] uses a grid-based measurement to differentiate and select the non-dominated solutions. In [34], a new farthest-candidate approach is proposed to replace the crowding distance mechanism in NSGA-II, and it is more effective to maintain the diversity of population.

There are also some other hybrid algorithms, such as NSGA-III [35] and the improved two-archive MaOEA [36]. In NSGA-III, a number of well-distributed reference points are initialized to guide the population along specific directions for maintaining good diversity [35]. The improved two-archive cooperation mechanism proposed in [36] respectively assigns two different indicators to the two archives in order to handle with the convergence and diversity separately. Other approaches based on reference vectors or preference information have been proposed and developed well [37-40].

\subsection{Dimensionality reduction}

In many-objective optimization, the dimensionality reduction technique aims to identify the potentially least amount of objectives to characterize the original optimization problem adequately [41-45]. Up to now, a variety 
of valuable dimensionality reduction approaches have been developed.

The first one is based on the idea of dominance relation preservation. Reference [41] proposes an effective objective reduction approach by preserving the dominance relations in the obtained PS. Specifically, given an objective $f \in F$ (i.e., the objective set), if the dominance relations between objectives keep constant when $f$ is deleted, then $f$ is regarded to be non-conflicting with the other members in $F$. Furthermore, a novel greedy algorithm is developed to resolve $\delta$-MOSS and k-EMOSS problems [41]. In [42], the conflict and non-conflict dominance relations between each pair of objectives are fist analyzed and then the non-conflicting ones are identified and amalgamated into one objective.

The second class is unsupervised feature selection. In [43], the objective correlation is analyzed, and then the objectives with more distance to each other are processed as the more conflicting ones. In this approach, the objective set is grouped into a set of neighborhood subsets with the size $q$ near each objective, and the neighborhood with the most compact structure is selected preferentially, whose central node is acquired and the corresponding neighbors are removed. Based on above paradigms, two algorithms have been developed to tackle $\delta$-MOSS and k-EMOSS problems [43].

The third one is the called Pareto corner search. Based on this principle, the algorithm in [44] explores only the corner segmentation of the PF, instead of searching for the entire PF. In this approach, the obtained non-dominated solutions are supposed to properly acquire the feature of the PF on each objective. Then the dimensionality reduction is accomplished with the assumption that it is acceptable to eliminating a redundant and an essential objective.

The forth type is machine learning based dimensionality reduction. The new approaches in $[45,46]$ take advantage of machine learning mechanism including principal component analysis (PCA) and maximum variance unfolding (MVU) to determine the priority of the dependences in the non-dominated solutions. Essentially, this scheme is based on the principle that high-dimensional solution structure can be well captured via minimizing the effect of noise and dependencies.

Furthermore, several nonlinear dimensionality reduction approaches have been developed, such as kernel PCA [47] and graph-based algorithm [48]. Reference [49] introduces a graph-based method into the MVU mechanism, within which the low-dimensional representation is tackled by gradually unfolding the high-dimensional information manifold. In this method, the unfolding is accomplished according to the Euclidean distances between points while the distances, with the preservation of distances and angles between adjacent points. 


\subsection{Surrogate models}

Recently many surrogate models have been employed to assist EAs as state-of-the-art search strategies [50-54]. For example, reference [51] proposes a surrogate model-aware search mechanism for medium scale computationally optimization problems, i.e., 20-50 decision variables, and a comparative study about surrogate-assisted multi-objective EA framework is conducted in [53]. These surrogate-assisted algorithms are able to effectively seek multiple optima and reduce the number of function evaluations by using information provided by surrogate models, e.g., Kriging methods [55-58]. These surrogate models may need additional computation costs and high usage of memory. It is stressed that these methods are increasingly useful as the problem complexity increases, because the computational cost caused by Kriging models is much less than that for function evaluations $[59,60]$. Hence, the surrogate models have significant potential to assist the dimensionality reduction scheme for many-objective optimization.

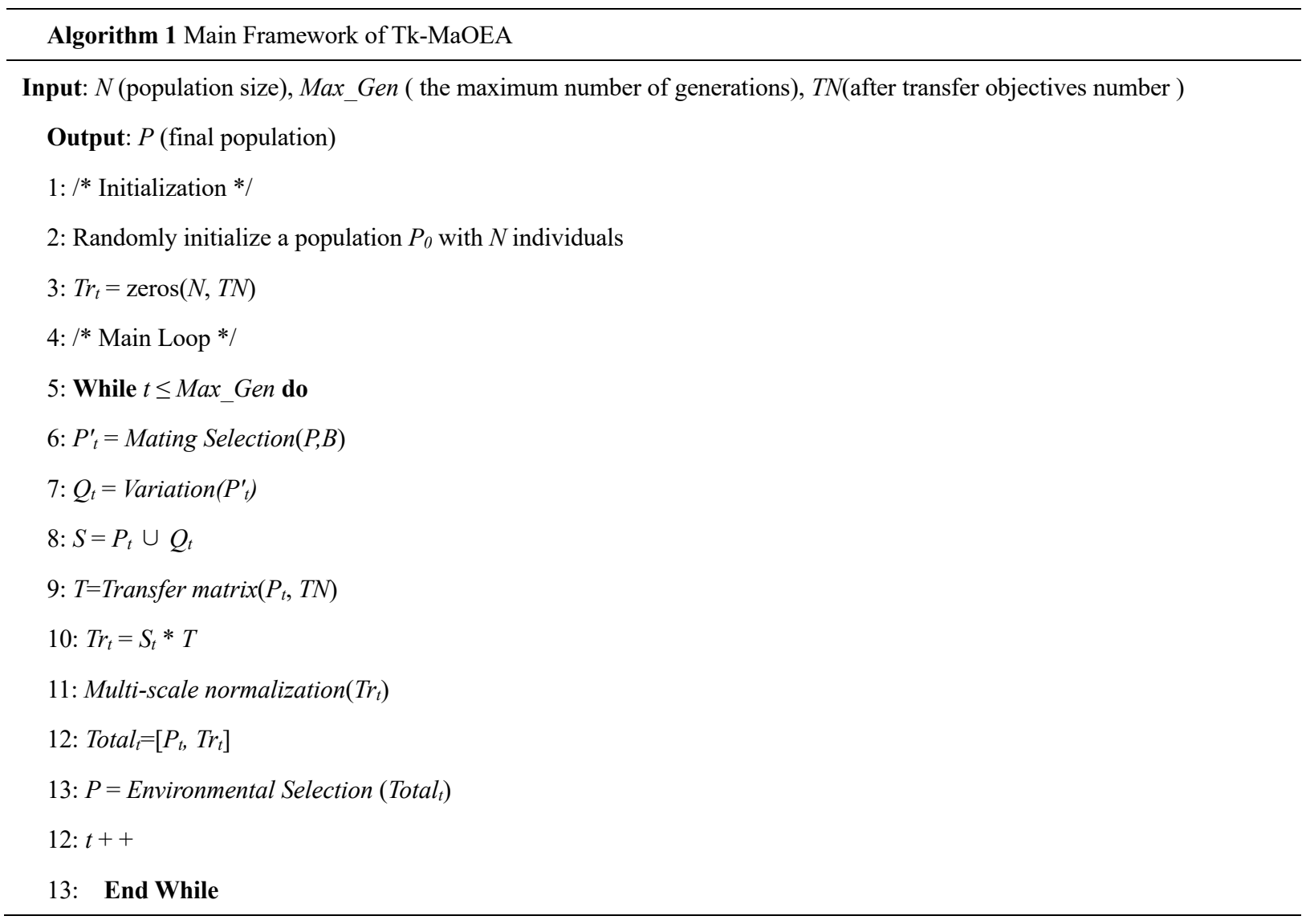

\section{Proposed algorithm}

\subsection{Basic idea}

Our approach uses transfer matrix based space reduction to drive the population to move in a relatively low 
dimensional search space, and then utilizes Kriging-assisted mechanism for each objective to enhance the exploration ability, based on the Latin hypercube sampling (LHS) method. In order to retain fast convergence as well as even distribution of the solutions, the farthest-candidate selection (FCS) is incorporated based on the fast non-dominated sorting approach in the environmental selection. As shown in Algorithm 1, the main framework of Tk-MaOEA is composed of the following components. First, in the initialization, $N$ individuals are initialized randomly to form a parent population (Lines 1-2 in Algorithm 1). Second, a binary tournament strategy is adopted to select solutions from the parent population to generate an offspring population $Q$ with $N$ individuals using a variation operation (Lines 6-8 in Algorithm 1). The variation operation employs conventional crossover and mutation used in [10]. Then, the combined population is transformed via transfer matrix to low dimensional objective space (Lines 9-12 in Algorithm1). Finally, $N$ solutions are selected from the combined population in the environmental selection procedure (Line 10 in Algorithm 1). In this procedure, the FCS method is employed to select elite individuals to maintain diversity of solutions for the next generation. These procedures repeat until a termination condition is me. The following subsections will show their details.

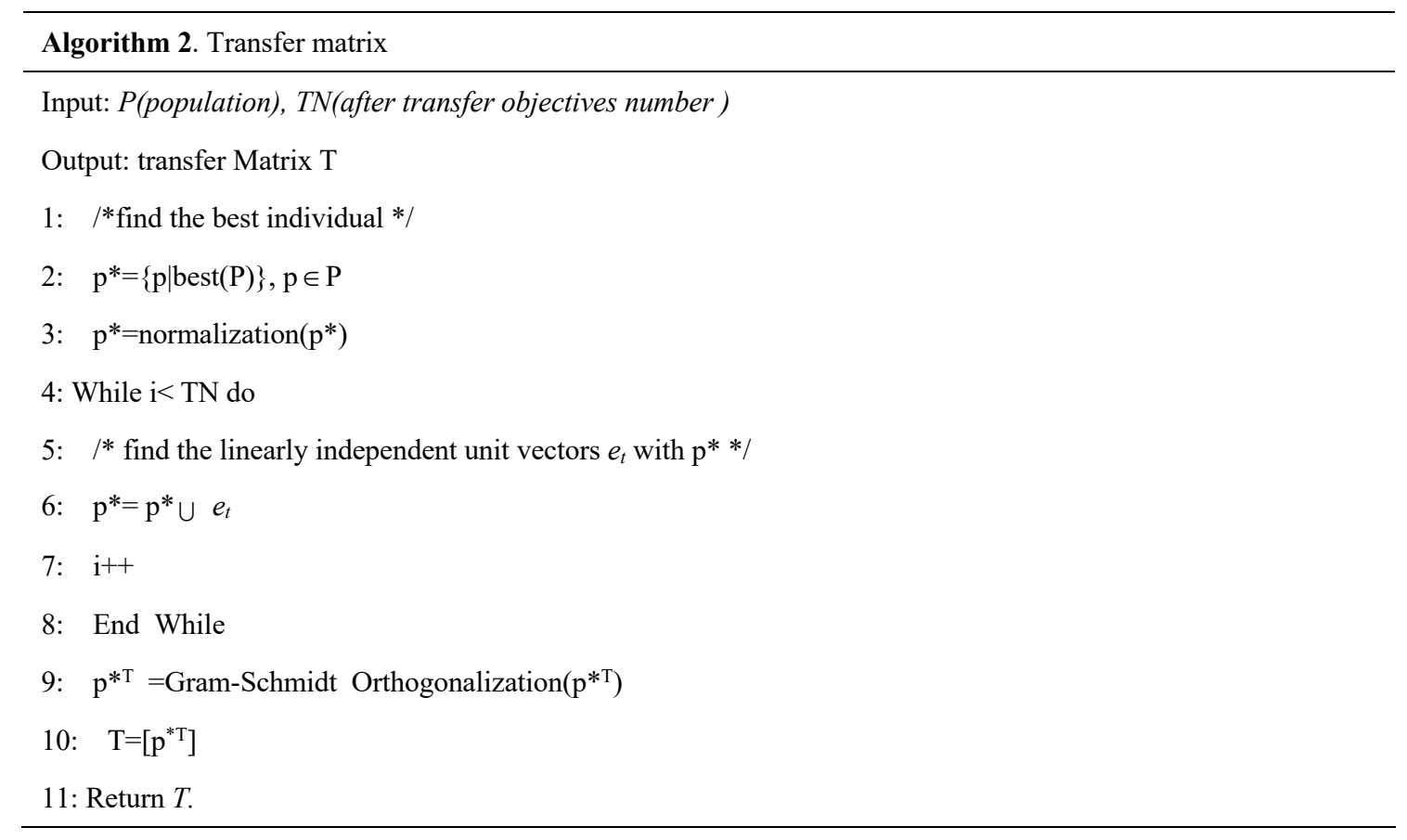

\subsection{Transfer Matrix}

Algorithm 2 shows the main principle of transfer matrix. For each generation, the best $P_{b e s t}$ is firstly selected (Lines 1-3 in Algorithm2). Then, the $T N-1$ linearly independent unit vectors are determined according to the $P_{b e s t}$ (Lines 4--7 in Algorithm2). As a result, the transfer matrix is constructed to makes up with $T N$ linearly independent column vectors. Next, the Gram-Schmidt Orthogonalization method is adopted for the 
orthogonalization of each column vector in the matrix, as shown in Algorithm 3. Accordingly, the first column in the transfer matrix is the best individual direction and other columns are the orthogonal direction with best individual, and each column is an unit. Theoretically, this transfer matrix can guide the other members in the population to learn from the best individual (refer to the proof of Theorem1 and Theorem 2 in Appendix). That is, the map lengths in the best individual direction and in the vertical individual direction can be used in the transfer learning. Therefore, a large number of objectives can be represented by a relatively small number of objectives, in virtue of the map length in the best individual direction and the map length of the other vertical individual direction.

In Euclidean space, it is desired that linearly independent vectors are transformed to orthogonal vectors. For this purpose, the Gram-Schmidt Orthogonalization (GSO) operation is devised as shown in Algorithm 3. First, each vector in the orthogonal vector group should be normalized (Lines 2 and 5 in Algorithm 3). Next, the GSO operation is implemented by using a linear combination of the inner product (Line 4 in Algorithm 3). Finally, the orthogonalized vector group is obtained, which will play a positive role on the algorithm.

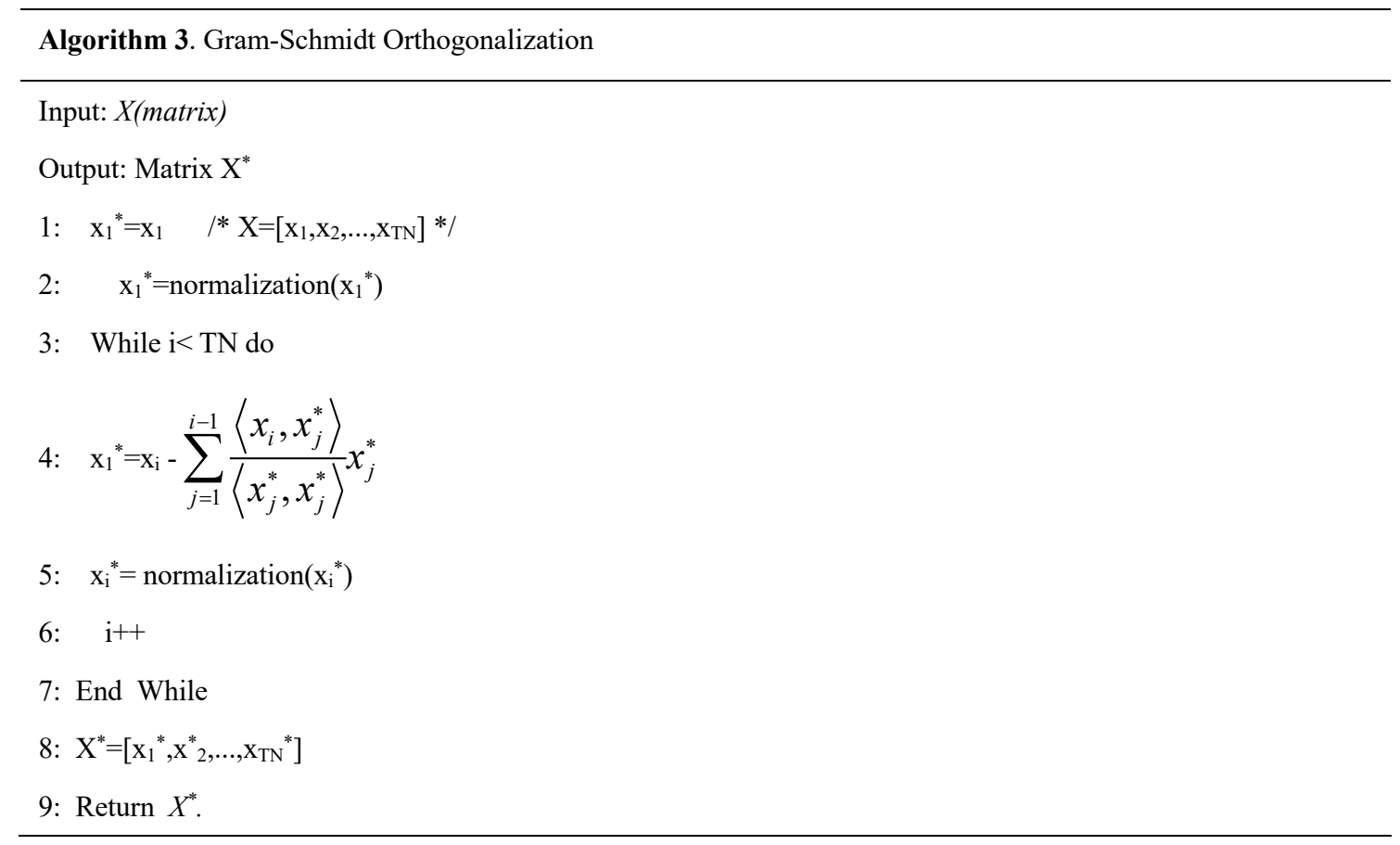

\subsection{Multi-scale nomination}

In the algorithm, it is desired to map them from a scaled objective space onto a normalized objective space. Note that, in many MaOPs such as WFG problems [50] and scaled DTLZ problems [51], their objective values are usually scaled disparately. In this case, the conventional normalization will generate a set of distorted solutions, whose spatial distribution is not consistent with the original ones. Therefore, as suggested in [52], 
instead of normalizing the objectives, we use the Schur product to translate $f_{i}^{*}(x)$ to $F_{i}(\mathrm{x})$ according to the boundary range of the objective values, as

$$
F_{i}(x)=\frac{f_{i}{ }^{\prime}(x) \circ\left(z^{\text {nad }}-z^{*}\right)}{\left\|f_{i}{ }^{\prime}(x) \circ\left(z^{\text {nad }}-z^{*}\right)\right\|}
$$

where $f_{i}^{\prime}(x)=f(x)-z_{i}^{m i n}$ is the $i$ th translated objective value, $z_{i}^{\min }$ and $z_{i}^{\max }$ are the $i$ th ideal point and the $i$ th nadir point, respectively. The binary operator $\circ$ denotes the Schur product, which takes two matrices of the same dimensions, and produces another matrix where each element is the product of elements of the original two matrices.

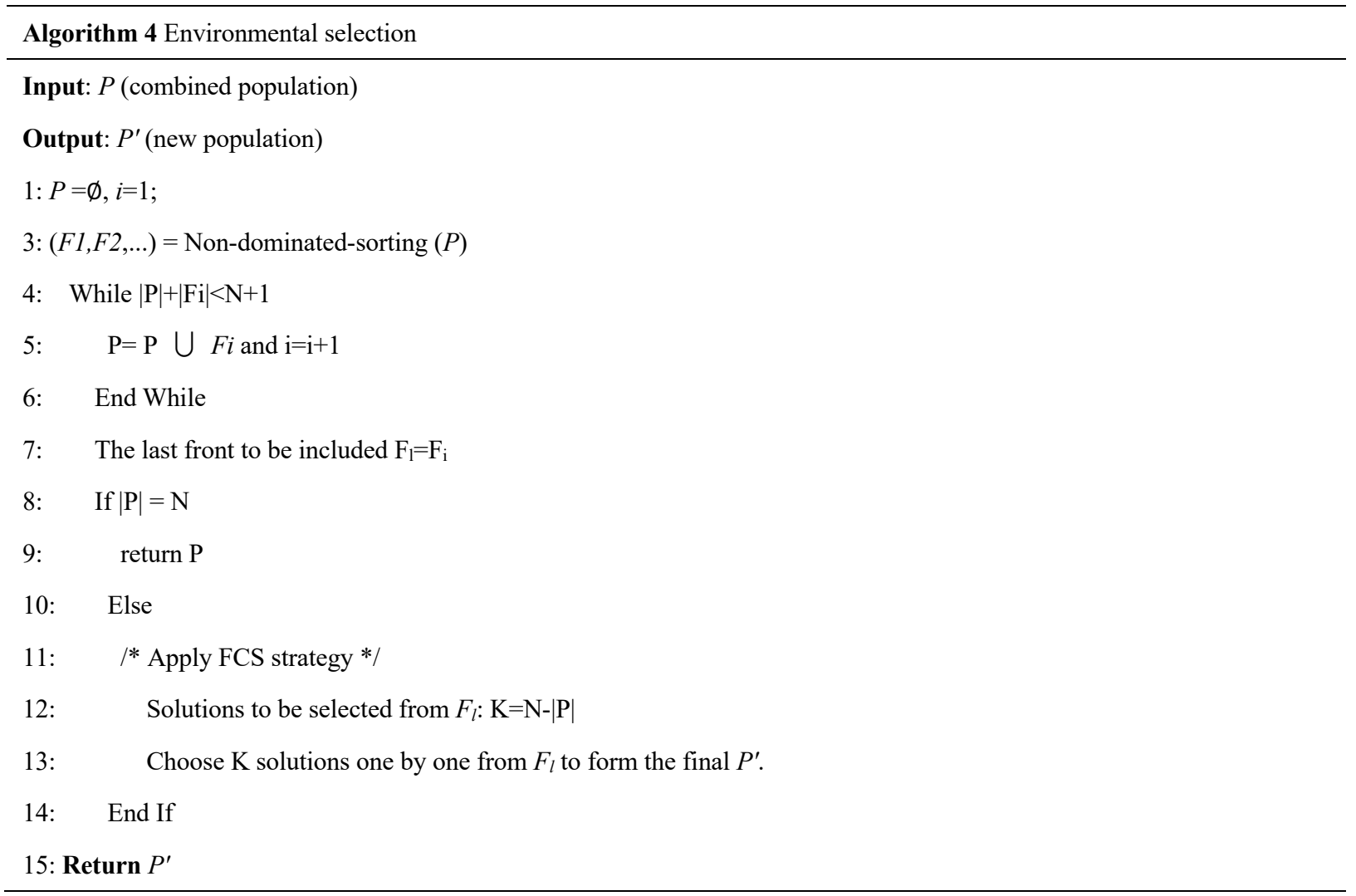

\subsection{Environmental selection}

Algorithm 4 illustrates the framework of environmental selection. Intuitively, this framework takes into consideration both the convergence and diversity of solutions, which are obtained by the Kriging-assisted mechanism and the FCS method, respectively. First, the traditional fast nondominated sorting is utilized to divide current solutions into different layers, and then the last layer $F_{l}$ is determined (lines 3-6 in Algorithm 4). If the population size is equal to $N$, then return $P$. Otherwise, $\mathrm{K}(\mathrm{N}-|\mathrm{P}|)$ solutions from $F_{l}$ are selected into $P$ one by one by using the called FCS approach (lines 11-13 in Algorithm 4), as presented as below in details.

\subsubsection{The FCS approach}


In Tk-MaOEA, instead of the traditional crowded distance method [10], an improved selection approach FCS, is devised, as suggested in [64]. Its main procedures is shown in Algorithm 5. In principle, the unselected individuals with farthest Euclidean distance from current selected solutions are selected preferentially as candidates. Specifically, in order to select $K$ elite individuals from the population, the boundary individuals with the smallest and largest fitness values are selected into the group of selected individuals (Lines 1-4 in Algorithm5). Then, the Euclidean distance between each solution and unselected ones are calculated and the minimum values of Euclidean distance are memorized (Lines 5-7 in Algorithm5). Finally, the farthest solutions are selected into $S_{\text {accept }}$ (Lines 8-11 in Algorithm5).
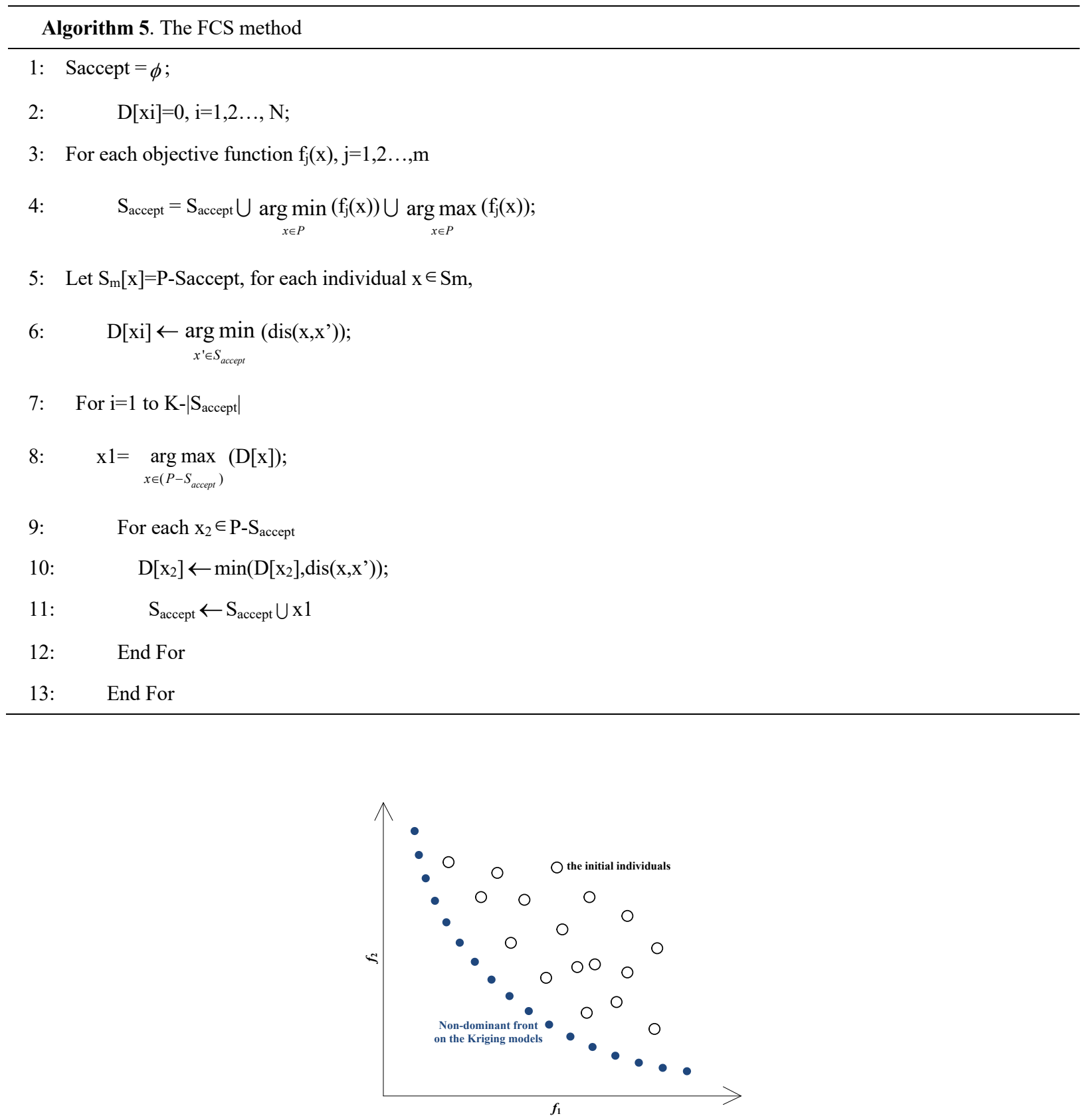

Fig. 1. Reference point definition using a ideal point of solutions on the Kriging models 


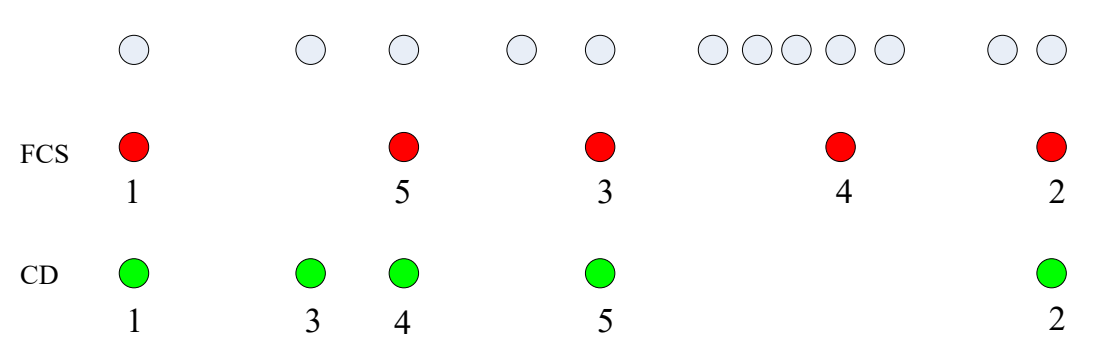

Fig. 2. Illustration of solutions selection by FCS and CD

The aim of the FCS method is to solve difficulties encountered by the crowded distance (CD) mechanism in particular situations where the solutions are not well-distributed. Take an example to illustrate, Fig. 2 shows 12 solutions to be proceeded by optimizers, and most of them are very close to each other whereas the others are not. In this case, optimizers need to select 5 solutions from 12 solutions. These selection results obtained by FCS and CD are identified by red and green, respectively. It is apparent that the spread of solutions obtained by FCS is significantly better than that obtained by $\mathrm{CD}$. This is because in the $\mathrm{CD}$ selection, the solution with a high density has a low chance to be selected, which may damage the spread of selected solutions. Fortunately, the FCS method can avoid this dilemma by using the principle of best-candidate sampling theory.

\subsubsection{Enhanced objective optimization based on Kriging model}

In Tk-MaOEA, the Kriging model is used for each objective function when the initial population is generated. Especially, following the approach in [65], the Kriging model is constructed by interpolating a number of uniformly-distributed individuals, initialized by Latin hypercube sampling (LHS) method [65]. Then, in the environmental selection process, the preferred solutions are selected from the Kriging model according to the estimated objective functions, as shown in Fig.1.

The ordinary Kriging model represents the unknown function $f(\mathrm{x})$, which is formulated as

$$
f(x)=a(x)+b(x)
$$

where $\mathrm{x}$ is an $\mathrm{m}$-dimensional decision vector, $\mathrm{a}(\mathrm{x})$ is a global model, and $\mathrm{b}(\mathrm{x})$ is a Gaussian process with $N\left(0, \sigma^{2}\right)$, which represents a local error with the global model. The correlation between $\mathrm{b}\left(\mathrm{x}_{\mathrm{i}}\right)$ and $\mathrm{b}\left(\mathrm{x}_{\mathrm{j}}\right)$ is strongly correlated to the distance between $\mathrm{x}_{\mathrm{i}}$ and $\mathrm{x}_{\mathrm{j}}$. Here, we use the Gaussian function with a weighted distance to define the correlation as

$$
\operatorname{Corr}\left(b\left(x_{i}\right), b\left(x_{j}\right)\right)=\exp \left(-\sum_{k=1}^{m} \omega^{k}\left(x_{i}^{k}-x_{j}^{k}\right)^{2}\right)
$$

where $\omega^{k}\left(0 \leq \omega^{k}<\infty\right)$ is the weight factor of the $k$ th element of an m-dimensional weight vector $\omega$. These weights maintain the anisotropy of the Kriging model and improve its accuracy. The predictor and uncertainty of 
the Kriging are expressed as

$$
\begin{aligned}
\tilde{f}(x) & =\tilde{b}(x)+r(x)^{T} R^{-1}(f-\tilde{b}) \\
v^{2}(x) & =\tilde{\sigma}^{2}\left(1-r(x)^{T} R^{-1} r(x)+\frac{\left(1-1^{T} R^{-1} r(x)\right)^{2}}{1^{T} R^{-1} 1}\right)
\end{aligned}
$$

where $\tilde{b}(x)$ is the approximated value of $b(x), R$ expresses the $n \times n$ matrix whose $(i, j)$ element is $\operatorname{Corr}\left(b\left(x_{i}\right), b\left(x_{j}\right)\right), \mathrm{r}(\mathrm{x})$ is an $\mathrm{n}$-dimensional vector whose $i$ th element is $\operatorname{Corr}\left(b\left(x_{i}\right), b\left(x_{j}\right)\right)$, and then $f$ and $\tilde{b}$ are formulized as follows when there are $n$ solutions

$$
\begin{gathered}
f=\left(f\left(\mathrm{x}^{1}\right) \ldots f\left(\mathrm{x}^{n}\right)\right)^{T} \\
\tilde{b}=\left(\tilde{b}\left(x^{1}\right) \ldots \tilde{b}\left(x^{n}\right)\right)^{T}
\end{gathered}
$$

and $\omega, \tilde{b}(x)$ and $\tilde{\sigma}^{2}$ (approximated $\sigma^{2}$ ) are the unknown parameters in the Kriging model. By maximizing the likelihood function, the unknown parameters are obtained [65].

Based on the Kriging model, the EI value, which is the expected objective function improvement from the current non-domination solution, is calculated according to the improvement value $I(x)$, expressed as

$$
\begin{aligned}
& I(\mathrm{x})=\max \left(f_{\text {ref }}-f, 0\right) \\
& E I(\mathrm{x})=\int_{-\infty}^{f_{\text {ref }}}\left(f_{\text {ref }}-f\right) \lambda(f) d f
\end{aligned}
$$

where $\lambda$ is the probability of $f$, whose density function is $N\left(\tilde{f}(x), v^{2}(x)\right), f_{\text {ref }}$ is the reference value of $f$, i.e., minimum value of $f(x)$. Accordingly, $E I(x)$ can be treated as the approximated value of the objective function.

Finally, the Kriging model is easily incorporated and implemented on each objective function in Tk-MaOEA.

\section{Experimental results}

In this section, the experimental study is conducted to evaluate the performance of the proposed Tk-MaOEA. Tk-MaOEA is benchmarked against a set of test functions including DTLZs [70] and WFGs[61], with several popular MaOEAs, namely MOEA/D [20], NSGA-III [35], MOMBII [25] and VaEA [7]. These algorithms have been verified to be effective on MaOPs, and they can be grouped into three classes: 1) the reference points or weight vectors based algorithms (MOEA/D and NSGA-III), 2) indicator based algorithm (MOMBII) and 3) Pareto dominance based algorithm (VaEA). The principal description of MOEA/D, NSGA-III, and VaEA can be referred in Sections I or their original literature $[8,35,7]$. MOMBII, as a recently proposed indicator based algorithm, takes a less-computation indicator called $\mathrm{R} 2$ as the selection criterion, which essentially weakens the Pareto compatibility [66]. Detailed presentation can be referred in reference [66]. 


\subsection{Test Problems and Performance Measures}

The first 4 instances (DTZL1 to DTLZ4) are taken from DTLZ [70]. where the number of decision variables is set to $n=M+r-1$, where $M$ is the objective number, $r=5$ for DTLZ1, and $r=10$ for DTLZ2 to DTLZ4. The other 9 test instances (WFG1 to WFG9) are taken from WFG [61], where the number of decision variables is set to $n=k+l-1$. As recommended in [61], the distance-related parameter $l$ is set ot 10 and the position-related parameter $k$ is set to $4,10,7$, and 9 for test instances with $M=3,5,8,10$, respectively. The attributes of involved problems include separability or nonseparability, unimodality or multimodality, unbiased or biased parameters, and convex or concave geometries. In order to quantitatively evaluate the performance of our proposed algorithm, two performance metrics are adopted: 1) convergence metric-IGD metric [67]; 2) hypervolume metric- Hv [57]. The further information about the two performance metrics can be referred in [67, 68]. Note that, as stated in [67], the number of reference points for computing IGD should be large enough so as to cover the complete PF as well as possible. Thus, the numbers of divisions for different numbers of objectives for DTLZs and WFGs are respectively listed in Table 1 and Table 2, where the last column shows the number of reference points for the problems. In addition, in order to indentify the significance of performance difference between those results obtained by Tk-MaOEA and its counterparts, Wilcoxon's rank sum test [69] is applied to obtained results with a level of significance $\mathrm{a}=0.05$.

Table 1 Number of reference points for DTLZs

\begin{tabular}{cccc}
\hline$M$ & $\mathrm{~h} 1(P)$ & $\mathrm{h} 2$ & Number of reference points \\
\hline 3 & 25 & - & 351 \\
5 & 13 & - & 2380 \\
8 & 7 & 6 & 5148 \\
10 & 6 & 5 & 7007 \\
\hline
\end{tabular}

Table 2 Number of reference points for WFGs

\begin{tabular}{ccccc}
\hline$M$ & WFG1 & WFG2 & WFG3 & WFG4-9 \\
\hline 3 & 421 & 148 & 5000 & 351 \\
5 & 2801 & 1601 & 17000 & 2380 \\
8 & 5464 & 4690 & 15000 & 5148 \\
10 & 20705 & 13634 & 26000 & 7007 \\
\hline
\end{tabular}

\subsection{Experimental Configuration}

The recommended parameter values for the algorithms that have obtained the best performance are configured as below. 
1) Population size: for MOEA/D and NSGA-III, the population size is set empirically according to the simplex-lattice design factor $H$ together with the objective number $M$. For VaEA, as recommended in [7], its population size keeps the same as that of NSGA-III. For the other two algorithms, Tk-MaOEA and MOMBII, the population sizes are set to the same as that of NSGA-III and MOEA/D, with respect to different objective numbers $M$.

2) Crossover and mutation: The SBX and polynomial mutation are used and the distribution indexes of crossover and mutation are set to $n_{c}=20$ and $n_{m}=20$, respectively. The crossover probability $p c=1.0$ and mutation probability $\mathrm{pm}=1 / D$, where $D$ is the number of decision variables.

3) Number of runs and termination condition: Each algorithm is performed for 20 independent runs on each test instance and the maximum function evaluations (MFEs) is set to 400000. For VaEA, the termination condition can be determined by $G \max =\operatorname{MFE} / N$, where $N$ is the population size.

4) Other parameters: For MOEA/D, the Tchebycheff approach is used with neighborhood range set to $\mathrm{N} / 10$ where $N$ is the population size. For MOMBII, involved parameters are set as $\varepsilon=1 e-3$ and $\alpha=0.5$. For Tk-MaOEA and VaEA, their parameters are set to the same as that of NSGA-III [10].

\subsection{Results and Analysis}

The experimental results of all algorithms over 3-, 5-, 8-, 10-objective test benchmarks are given in Table 3, Table 4 and Table 5. In these tables, the mean and standard deviation (SD) values in terms of the HV and IGD metrics obtained by the MaOEAs over 20 independent runs are reported. The significance of difference between Tk-MaOEA and the compared algorithms is evaluated by Wilcoxon's rank sum test.

\subsubsection{Results in terms of HV metric}

As shown in Table 3, Tk-MaOEA is the most effective performer, which achieves the first or second ranks on most of DTLZ test instances. NSGA-III and VaEA also obtain satisfactory performance. Specifically, NSGA-III obtains the first ranks on 8-, 10-objective DTLZ1, 5- and 8-objective DTLZ2, while VaEA is ranked the first on 5-objective DTLZ3 and 8-objective DTLZ4. MOMBII and MOEA/D obtain similar performance, doing well on low-dimensional instances, such as 3-objective DTLZ1 and DTLZ4. In fact, the statistical results in terms of IGD values for all the algorithms are close to each other.

As for the WFG instances, it can be observed from Table 4 that Tk-MaOEA performs very powerfully, retaining the first or second ranks on most of test instances. As shown, both Tk-MaOEA and NSGA-III perform powerfully, exhibiting an obvious superiority to other involved algorithms on the majority of the WFG test instances. Specifically, Tk-MaOEA obtains the first and second ranks in terms of HV values on 17 and 6 out of 
the 36 test instances, respectively. At the same time, NSGA-III obtains 10 first-rank results on all test instances while MOMBII and VaEA also retains the fist ranks on 6 out of the 36 test instances. On 8-objective WFG3 and 8-objective WFG8, MOEA/D does very competently, ranked the first. For 5-objective WFG3, 3-objective WFG7 and 8-objective WFG7, VaEA performs only slightly better than Tk-MaOEA.

For WFG1, NSGA-III performs the best, but just only a little better than Tk-MaOEA. In fact, the difference between their mean results are very close. On WFG2, Tk-MaOEA does the best on 8- and 10-objective instances while NSGA-III performs best on 5-objective instances. In fact, on 10-objecitve WFG2 instance, the performance of Tk-MaOEA is significantly better than that of NSGA-III. It should be stressed that the performance of Tk-MaOEA is not deteriorated when the number of objectives increases, unlike other algorithms. MOMBII also achieves the best performance on 3-objective WFG2. On WFG3, all the involved algorithms except NSGA-III obtain similar performance on 3- and 8-objective instances. Tk-MaOEA obtains the first rank on the 10-objective instance, while MOEA/D also performs very powerfully on this instance. As the number of objectives becomes large (i.e., $\mathrm{M}=8$ and $\mathrm{M}=10$ ), the performance of MOMBII seem somewhat worse than that of NSGA-III and Tk-MaOEA .

For WFG4, whose PF has many local optima to be difficult to optimized. Tk-MaOEA obtains the first or second results on 3 out of the 4 test instances, which are 8- and 10-objective, and MOMBII also finds the best result on the 3-objective instance, but struggle on the higher-dimensional cases. For WFG5, Tk-MaOEA performs most powerfully, ranked first on most of the test instances. For nonseparable WFG6, Tk-MaOEA obtains a satisfactory performance, only worse than that of NSGA-III on 8-objective instance. Similar observation are obtained on WFG7, which is the separable and unimodal problem, Tk-MaOEA does better or at least comparably to VaEA, yet superior to other algorithms. On nonseparable WFG8, similar to the WFG6 case, Tk-MaOEA is the best performer on most of test instances, and only slightly worse than NSGA-III on 3-objecitve instances. On WFG9, NSGA-III obtains satisfactory performance, only worse than Tk-MaOEA on the 3- and 10-objective instances, while VaEA does best on 8-objective instance. These results show the effectiveness of the proposed strategies in Tk-MaOEA 
Table 3 Mean and standard deviation results of HV obtained by Tk-MaOEA, MOEA/D, MOMBII, VaEA and NSGA-III on DTLZs1-4 (The best items are in bold).

\begin{tabular}{|c|c|c|c|c|c|c|}
\hline Problem & M & Tk-MaOEA & MOEA/D & MOMBII & VaEA & NSGA-III \\
\hline \multirow{4}{*}{ DTLZ1 } & 3 & $8.793 \mathrm{e}-01 / 4.175 \mathrm{e}-02$ & $8.417 \mathrm{e}-01 / 8.374 \mathrm{e}-06+$ & $8.416 \mathrm{e}-01 / 3.096 \mathrm{e}-05+$ & $8.300 \mathrm{e}-01 / 4.906 \mathrm{e}-03+$ & $8.413 \mathrm{e}-01 / 2.375 \mathrm{e}-04+$ \\
\hline & 5 & $8.793 \mathrm{e}-01 / 1.416 \mathrm{e}-01$ & $9.704 \mathrm{e}-01 / 8.132 \mathrm{e}-05-$ & $9.706 e-01 / 1.117 e-04-$ & $8.833 \mathrm{e}-01 / 3.778 \mathrm{e}-02-$ & $9.705 e-01 / 4.808 \mathrm{e}-05-$ \\
\hline & 8 & $9.752 \mathrm{e}-01 / 1.003 \mathrm{e}-02$ & $9.753 \mathrm{e}-01 / 8.447 \mathrm{e}-04-$ & $6.285 \mathrm{e}-01 / 2.276 \mathrm{e}-01+$ & $8.799 \mathrm{e}-01 / 2.585 \mathrm{e}-02+$ & $9.896 \mathrm{e}-01 / 7.156 \mathrm{e}-04-$ \\
\hline & 10 & $9.194 \mathrm{e}-01 / 3.035 \mathrm{e}-02$ & 9.951e-01/4.759e-04- & $7.575 \mathrm{e}-01 / 2.841 \mathrm{e}-02+$ & $9.280 \mathrm{e}-01 / 5.252 \mathrm{e}-02-$ & $9.983 e-01 / 1.817 e-02-$ \\
\hline \multirow{4}{*}{ DTLZ2 } & 3 & $5.233 \mathrm{e}-01 / 5.110 \mathrm{e}-02$ & $5.596 \mathrm{e}-01 / 1.265 \mathrm{e}-06-$ & $5.595 \mathrm{e}-01 / 5.118 \mathrm{e}-05-$ & $5.558 \mathrm{e}-01 / 1.409 \mathrm{e}-03-$ & $5.596 \mathrm{e}-01 / 2.708 \mathrm{e}-06-$ \\
\hline & 5 & $7.075 \mathrm{e}-01 / 8.613 \mathrm{e}-02$ & $7.743 \mathrm{e}-01 / 4.455 \mathrm{e}-04-$ & $7.745 \mathrm{e}-01 / 3.373 \mathrm{e}-04-$ & $7.634 \mathrm{e}-01 / 1.895 \mathrm{e}-04-$ & 7.746e-01/2.899e-05- \\
\hline & 8 & $8.133 \mathrm{e}-01 / 1.053 \mathrm{e}-01$ & $8.849 \mathrm{e}-01 / 6.477 \mathrm{e}-04-$ & $7.832 \mathrm{e}-01 / 2.307 \mathrm{e}-03+$ & $8.847 \mathrm{e}-01 / 8.236 \mathrm{e}-03-$ & $8.942 \mathrm{e}-01 / 1.436 \mathrm{e}-01-$ \\
\hline & 10 & $9.552 \mathrm{e}-01 / 1.146 \mathrm{e}-03$ & $9.371 \mathrm{e}-01 / 7.707 \mathrm{e}-05+$ & $7.626 \mathrm{e}-01 / 3.253 \mathrm{e}-05+$ & $9.150 \mathrm{e}-01 / 2.980 \mathrm{e}-03+$ & $7.884 \mathrm{e}-01 / 3.131 \mathrm{e}-03+$ \\
\hline \multirow{4}{*}{ DTLZ3 } & 3 & $7.908 \mathrm{e}-01 / 1.837 \mathrm{e}-02$ & $5.537 \mathrm{e}-01 / 8.033 \mathrm{e}-04+$ & $5.561 \mathrm{e}-01 / 4.224 \mathrm{e}-03+$ & $5.511 \mathrm{e}-01 / 8.692 \mathrm{e}-03+$ & $5.558 \mathrm{e}-01 / 3.306 \mathrm{e}-03+$ \\
\hline & 5 & $6.356 \mathrm{e}-01 / 8.989 \mathrm{e}-02$ & $7.661 \mathrm{e}-01 / 9.294 \mathrm{e}-03-$ & $7.720 \mathrm{e}-01 / 1.232 \mathrm{e}-03-$ & $7.954 \mathrm{e}-01 / 2.764 \mathrm{e}-01-$ & 7.728e-01/4.731e-04- \\
\hline & 8 & $4.122 \mathrm{e}-01 / 5.098 \mathrm{e}-02$ & $1.247 \mathrm{e}-01 / 4.035 \mathrm{e}-02+$ & $4.392 \mathrm{e}-01 / 1.790 \mathrm{e}-03-$ & $1.568 \mathrm{e}-01 / 1.156 \mathrm{e}-02+$ & $4.373 \mathrm{e}-01 / 6.184 \mathrm{e}-01-$ \\
\hline & 10 & $5.647 \mathrm{e}-01 / 5.778 \mathrm{e}-03$ & $5.568 \mathrm{e}-01 / 5.245 \mathrm{e}-01+$ & $4.037 \mathrm{e}-01 / 2.316 \mathrm{e}-02+$ & $1.687 \mathrm{e}-01 / 5.231 \mathrm{e}-01+$ & $3.569 \mathrm{e}-01 / 3.256 \mathrm{e}-01+$ \\
\hline \multirow{4}{*}{ DTLZ4 } & 3 & $5.407 \mathrm{e}-01 / 7.038 \mathrm{e}-02$ & $4.472 \mathrm{e}-01 / 1.497 \mathrm{e}-01+$ & $5.595 \mathrm{e}-01 / 1.653 \mathrm{e}-05-$ & $5.548 \mathrm{e}-01 / 4.160 \mathrm{e}-03-$ & $4.499 \mathrm{e}-01 / 1.551 \mathrm{e}-01+$ \\
\hline & 5 & $7.813 e-01 / 1.279 e-01$ & $7.268 \mathrm{e}-01 / 6.589 \mathrm{e}-02+$ & $6.452 \mathrm{e}-01 / 1.824 \mathrm{e}-01+$ & $7.670 \mathrm{e}-01 / 4.093 \mathrm{e}-03+$ & 7.188e-01/7.797e-02+ \\
\hline & 8 & $8.129 \mathrm{e}-01 / 2.198 \mathrm{e}-02$ & $7.377 \mathrm{e}-01 / 6.152 \mathrm{e}-02+$ & $8.338 \mathrm{e}-01 / 7.319 \mathrm{e}-02-$ & $8.861 \mathrm{e}-01 / 2.270 \mathrm{e}-03-$ & $7.218 \mathrm{e}-01 / 5.799 \mathrm{e}-03+$ \\
\hline & 10 & $9.849 \mathrm{e}-01 / 1.461 \mathrm{e}-01$ & $7.585 \mathrm{e}-01 / 2.204 \mathrm{e}-03+$ & $8.313 \mathrm{e}-01 / 1.877 \mathrm{e}-03+$ & $9.146 \mathrm{e}-01 / 6.859 \mathrm{e}-05+$ & $7.872 \mathrm{e}-01 / 3.563 \mathrm{e}-02+$ \\
\hline
\end{tabular}


Table 4 Mean and standard deviation results of HV obtained by Tk-MaOEA, MOEA/D, MOMBII, VaEA and NSGA-III on WFGs 1-9 (The best items are in bold).

\begin{tabular}{|c|c|c|c|c|c|c|}
\hline Problem & $\mathrm{M}$ & Tk-MaOEA & MOEA/D & MOMBII & VaEA & NSGA-III \\
\hline \multirow{4}{*}{ WFG1 } & 3 & $9.224 \mathrm{e}-01 / 5.801 \mathrm{e}-03$ & $9.180 \mathrm{e}-01 / 1.751 \mathrm{e}-03+$ & $9.205 \mathrm{e}-01 / 6.694 \mathrm{e}-04+$ & $9.391 \mathrm{e}-01 / 2.087 \mathrm{e}-04-$ & $9.447 \mathrm{e}-01 / 1.736 \mathrm{e}-03-$ \\
\hline & 5 & $9.402 \mathrm{e}-01 / 2.960 \mathrm{e}-02$ & $9.327 \mathrm{e}-01 / 1.469 \mathrm{e}-03+$ & $9.917 \mathrm{e}-01 / 8.952 \mathrm{e}-04-$ & $9.192 \mathrm{e}-01 / 3.843 \mathrm{e}-02+$ & 9.976e-01/1.067e-04- \\
\hline & 8 & $9.498 \mathrm{e}-01 / 2.281 \mathrm{e}-02$ & $8.741 \mathrm{e}-01 / 6.866 \mathrm{e}-02+$ & $8.294 \mathrm{e}-01 / 1.145 \mathrm{e}-01+$ & $9.471 \mathrm{e}-01 / 6.239 \mathrm{e}-02+$ & $9.995 \mathrm{e}-01 / 2.770 \mathrm{e}-04-$ \\
\hline & 10 & $9.230 \mathrm{e}-01 / 3.710 \mathrm{e}-02$ & $5.946 \mathrm{e}-01 / 4.291 \mathrm{e}-02+$ & $8.851 \mathrm{e}-01 / 7.744 \mathrm{e}-05+$ & $8.734 \mathrm{e}-01 / 5.009 \mathrm{e}-02+$ & $9.944 \mathrm{e}-01 / 7.769 \mathrm{e}-03-$ \\
\hline \multirow{4}{*}{ WFG2 } & 3 & $6.013 \mathrm{e}-01 / 3.745 \mathrm{e}-02$ & 9.156e-01/9.312e-04- & $9.463 \mathrm{e}-01 / 9.287 \mathrm{e}-04-$ & $9.256 \mathrm{e}-01 / 5.235 \mathrm{e}-03-$ & $9.312 \mathrm{e}-01 / 8.426 \mathrm{e}-04-$ \\
\hline & 5 & $7.872 \mathrm{e}-01 / 9.812 \mathrm{e}-04$ & $9.515 \mathrm{e}-01 / 6.935 \mathrm{e}-03-$ & $9.742 \mathrm{e}-01 / 2.560 \mathrm{e}-02-$ & $9.894 \mathrm{e}-01 / 1.167 \mathrm{e}-03-$ & $9.956 \mathrm{e}-01 / 1.273 \mathrm{e}-04-$ \\
\hline & 8 & $9.911 \mathrm{e}-01 / 2.720 \mathrm{e}-02$ & $9.021 \mathrm{e}-01 / 6.911 \mathrm{e}-03+$ & $8.935 \mathrm{e}-01 / 4.877 \mathrm{e}-02+$ & $9.857 \mathrm{e}-01 / 2.150 \mathrm{e}-03+$ & $9.908 \mathrm{e}-01 / 1.988 \mathrm{e}-03+$ \\
\hline & 10 & $9.963 \mathrm{e}-01 / 6.456 \mathrm{e}-03$ & $9.248 \mathrm{e}-01 / 1.394 \mathrm{e}-02+$ & $9.588 \mathrm{e}-01 / 2.715 \mathrm{e}-03+$ & $9.960 \mathrm{e}-01 / 1.192 \mathrm{e}-04+$ & $9.941 \mathrm{e}-01 / 2.000 \mathrm{e}-03+$ \\
\hline \multirow{4}{*}{ WFG3 } & 3 & $4.516 \mathrm{e}-01 / 3.306 \mathrm{e}-03$ & $3.626 \mathrm{e}-01 / 2.510 \mathrm{e}-03+$ & $4.029 \mathrm{e}-01 / 2.343 \mathrm{e}-03+$ & $3.684 \mathrm{e}-01 / 1.338 \mathrm{e}-02+$ & $3.886 \mathrm{e}-01 / 4.755 \mathrm{e}-03+$ \\
\hline & 5 & $1.883 \mathrm{e}-01 / 3.542 \mathrm{e}-02$ & $1.042 \mathrm{e}-01 / 4.313 \mathrm{e}-02+$ & $1.854 \mathrm{e}-01 / 2.220 \mathrm{e}-02+$ & $1.971 \mathrm{e}-01 / 6.574 \mathrm{e}-03-$ & $1.886 \mathrm{e}-01 / 7.917 \mathrm{e}-03 \approx$ \\
\hline & 8 & $8.362 \mathrm{e}-02 / 3.247 \mathrm{e}-03$ & $8.923 \mathrm{e}-02 / 1.123 \mathrm{e}-02-$ & $8.630 \mathrm{e}-02 / 3.709 \mathrm{e}-03-$ & $7.342 \mathrm{e}-02 / 1.705 \mathrm{e}-03+$ & $6.165 \mathrm{e}-02 / 3.932 \mathrm{e}-03+$ \\
\hline & 10 & $8.464 \mathrm{e}-02 / 1.4563 \mathrm{e}-02$ & $7.842 \mathrm{e}-02 / 1.842 \mathrm{e}-02+$ & $6.514 \mathrm{e}-02 / 1.412 \mathrm{e}-02+$ & $5.763 \mathrm{e}-02 / 1.618 \mathrm{e}-02+$ & $8.112 \mathrm{e}-02 / 1.573 \mathrm{e}-02+$ \\
\hline \multirow{4}{*}{ WFG4 } & 3 & $5.198 \mathrm{e}-01 / 2.600 \mathrm{e}-02$ & $5.450 \mathrm{e}-01 / 1.029 \mathrm{e}-03-$ & $5.595 \mathrm{e}-01 / 2.365 \mathrm{e}-04-$ & $5.511 \mathrm{e}-01 / 1.941 \mathrm{e}-03-$ & $5.591 \mathrm{e}-01 / 2.210 \mathrm{e}-04-$ \\
\hline & 5 & $7.637 \mathrm{e}-01 / 3.124 \mathrm{e}-02$ & $6.561 \mathrm{e}-01 / 1.345 \mathrm{e}-02+$ & $7.568 \mathrm{e}-01 / 1.136 \mathrm{e}-02+$ & $7.552 \mathrm{e}-01 / 4.417 \mathrm{e}-03+$ & 7.721e-01/1.394e-03- \\
\hline & 8 & $9.147 e-01 / 1.987 e-02$ & $3.351 \mathrm{e}-01 / 3.913 \mathrm{e}-03+$ & $6.681 \mathrm{e}-01 / 1.662 \mathrm{e}-01+$ & $8.697 \mathrm{e}-01 / 1.860 \mathrm{e}-02+$ & $8.816 \mathrm{e}-01 / 6.595 \mathrm{e}-04+$ \\
\hline & 10 & $9.070 e-01 / 3.626 e-02$ & $3.564 \mathrm{e}-01 / 7.592 \mathrm{e}-02+$ & $4.296 \mathrm{e}-01 / 1.039 \mathrm{e}-01+$ & $8.971 \mathrm{e}-01 / 2.591 \mathrm{e}-04+$ & $8.710 \mathrm{e}-01 / 9.506 \mathrm{e}-02+$ \\
\hline \multirow{4}{*}{ WFG5 } & 3 & $5.909 \mathrm{e}-01 / 9.890 \mathrm{e}-02$ & $5.087 \mathrm{e}-01 / 6.013 \mathrm{e}-04+$ & $5.059 \mathrm{e}-01 / 5.489 \mathrm{e}-03+$ & $5.156 \mathrm{e}-01 / 2.759 \mathrm{e}-04+$ & $5.184 \mathrm{e}-01 / 8.397 \mathrm{e}-05+$ \\
\hline & 5 & $7.887 e-01 / 3.057 e-02$ & $6.178 \mathrm{e}-01 / 2.998 \mathrm{e}-02+$ & $6.965 \mathrm{e}-01 / 2.422 \mathrm{e}-03+$ & $7.123 \mathrm{e}-01 / 3.946 \mathrm{e}-04+$ & $7.238 \mathrm{e}-01 / 6.525 \mathrm{e}-04+$ \\
\hline & 8 & $8.735 e-01 / 2.172 e-01$ & $3.657 \mathrm{e}-01 / 6.155 \mathrm{e}-02+$ & $4.718 \mathrm{e}-01 / 3.179 \mathrm{e}-01+$ & $8.178 \mathrm{e}-01 / 1.021 \mathrm{e}-03+$ & $8.235 \mathrm{e}-01 / 2.687 \mathrm{e}-03+$ \\
\hline & 10 & $8.125 \mathrm{e}-01 / 4.642 \mathrm{e}-02$ & $3.387 \mathrm{e}-01 / 4.715 \mathrm{e}-02+$ & $2.284 \mathrm{e}-01 / 1.667 \mathrm{e}-02+$ & $8.315 \mathrm{e}-01 / 5.996 \mathrm{e}-03-$ & 8.748e-01/2.691e-04- \\
\hline \multirow{4}{*}{ WFG6 } & 3 & $7.153 e-01 / 3.097 e-02$ & $4.719 \mathrm{e}-01 / 1.281 \mathrm{e}-02+$ & $4.864 \mathrm{e}-01 / 1.501 \mathrm{e}-02+$ & $4.989 \mathrm{e}-01 / 1.434 \mathrm{e}-02+$ & $5.142 \mathrm{e}-01 / 1.682 \mathrm{e}-02+$ \\
\hline & 5 & $7.020 e-01 / 1.299 e-02$ & $5.037 \mathrm{e}-01 / 1.170 \mathrm{e}-03+$ & $6.091 \mathrm{e}-01 / 2.726 \mathrm{e}-02+$ & $6.952 \mathrm{e}-01 / 2.995 \mathrm{e}-02+$ & $6.938 \mathrm{e}-01 / 2.098 \mathrm{e}-02+$ \\
\hline & 8 & $7.468 \mathrm{e}-01 / 2.537 \mathrm{e}-02$ & $2.451 \mathrm{e}-01 / 4.539 \mathrm{e}-02+$ & $7.245 \mathrm{e}-01 / 1.170 \mathrm{e}-01+$ & $8.160 \mathrm{e}-01 / 4.098 \mathrm{e}-02-$ & 8.296e-01/8.089e-02- \\
\hline & 10 & $8.815 \mathrm{e}-01 / 8.172 \mathrm{e}-03$ & $1.433 \mathrm{e}-01 / 5.667 \mathrm{e}-02+$ & $7.127 \mathrm{e}-01 / 1.283 \mathrm{e}-01+$ & $7.883 \mathrm{e}-01 / 1.877 \mathrm{e}-02+$ & $8.188 \mathrm{e}-01 / 2.407 \mathrm{e}-04+$ \\
\hline \multirow{2}{*}{ WFG7 } & 3 & $5.076 \mathrm{e}-01 / 8.466 \mathrm{e}-03$ & 5.338e-01/2.111e-03- & $5.592 \mathrm{e}-01 / 3.608 \mathrm{e}-05-$ & $5.605 e-01 / 3.228 \mathrm{e}-03-$ & $5.587 \mathrm{e}-01 / 4.260 \mathrm{e}-05-$ \\
\hline & 5 & $7.157 \mathrm{e}-01 / 1.232 \mathrm{e}-02$ & $5.470 \mathrm{e}-01 / 1.196 \mathrm{e}-03+$ & $7.741 \mathrm{e}-01 / 4.737 \mathrm{e}-04-$ & $7.605 \mathrm{e}-01 / 1.972 \mathrm{e}-03-$ & 7.813e-01/3.638e-04- \\
\hline
\end{tabular}




\begin{tabular}{|c|c|c|c|c|c|c|}
\hline & 8 & $8.288 \mathrm{e}-01 / 3.769 \mathrm{e}-02$ & $2.718 \mathrm{e}-01 / 7.078 \mathrm{e}-03+$ & $8.298 \mathrm{e}-01 / 7.905 \mathrm{e}-02 \approx$ & $8.782 \mathrm{e}-01 / 2.994 \mathrm{e}-03-$ & $8.223 \mathrm{e}-01 / 8.025 \mathrm{e}-02 \approx$ \\
\hline & 10 & $9.769 \mathrm{e}-01 / 3.904 \mathrm{e}-02$ & $2.117 \mathrm{e}-01 / 6.828 \mathrm{e}-02+$ & $5.479 \mathrm{e}-01 / 1.673 \mathrm{e}-02+$ & $9.131 \mathrm{e}-01 / 7.131 \mathrm{e}-03+$ & $9.140 \mathrm{e}-01 / 3.421 \mathrm{e}-02+$ \\
\hline \multirow{4}{*}{ WFG8 } & 3 & $4.685 \mathrm{e}-01 / 1.564 \mathrm{e}-03$ & $4.670 \mathrm{e}-01 / 2.009 \mathrm{e}-04 \approx$ & $4.515 \mathrm{e}-01 / 4.559 \mathrm{e}-03+$ & $4.653 \mathrm{e}-01 / 1.879 \mathrm{e}-03 \approx$ & $4.745 \mathrm{e}-01 / 1.505 \mathrm{e}-03-$ \\
\hline & 5 & $6.674 \mathrm{e}-01 / 2.764 \mathrm{e}-02$ & $2.734 \mathrm{e}-01 / 3.825 \mathrm{e}-02+$ & $4.641 \mathrm{e}-01 / 2.884 \mathrm{e}-03+$ & $6.230 \mathrm{e}-01 / 4.084 \mathrm{e}-03+$ & $6.589 \mathrm{e}-01 / 2.196 \mathrm{e}-03+$ \\
\hline & 8 & $7.125 \mathrm{e}-01 / 2.341 \mathrm{e}-02$ & 7.333e-01/1.221e-02- & $4.718 \mathrm{e}-01 / 3.947 \mathrm{e}-02+$ & $7.195 \mathrm{e}-01 / 1.273 \mathrm{e}-03 \approx$ & $6.611 \mathrm{e}-01 / 2.335 \mathrm{e}-02+$ \\
\hline & 10 & $7.854 \mathrm{e}-01 / 9.329 \mathrm{e}-02$ & $6.842 \mathrm{e}-01 / 5.619 \mathrm{e}-02+$ & $3.837 \mathrm{e}-01 / 6.862 \mathrm{e}-02+$ & $7.533 \mathrm{e}-01 / 1.922 \mathrm{e}-02+$ & $7.754 \mathrm{e}-01 / 9.837 \mathrm{e}-02+$ \\
\hline \multirow{4}{*}{ WFG9 } & 3 & $5.561 \mathrm{e}-01 / 2.200 \mathrm{e}-02$ & $5.075 \mathrm{e}-01 / 8.088 \mathrm{e}-03+$ & $5.075 \mathrm{e}-01 / 6.579 \mathrm{e}-03+$ & $5.254 \mathrm{e}-01 / 6.211 \mathrm{e}-04+$ & $5.406 \mathrm{e}-01 / 1.114 \mathrm{e}-03+$ \\
\hline & 5 & $6.430 \mathrm{e}-01 / 3.995 \mathrm{e}-02$ & $6.082 \mathrm{e}-01 / 2.899 \mathrm{e}-02+$ & $5.737 \mathrm{e}-01 / 1.151 \mathrm{e}-02+$ & $7.079 \mathrm{e}-01 / 3.113 \mathrm{e}-04-$ & 7.509e-01/9.322e-02- \\
\hline & 8 & $5.259 \mathrm{e}-01 / 4.295 \mathrm{e}-02$ & $2.861 \mathrm{e}-01 / 7.858 \mathrm{e}-02+$ & $8.067 e-01 / 8.315 e-03-$ & $7.928 \mathrm{e}-01 / 2.112 \mathrm{e}-03-$ & 7.237e-01/1.069e-01- \\
\hline & 10 & $9.393 e-01 / 5.137 e-02$ & $1.585 \mathrm{e}-01 / 1.369 \mathrm{e}-02+$ & $1.459 \mathrm{e}-01 / 3.442 \mathrm{e}-02+$ & $7.911 \mathrm{e}-01 / 1.653 \mathrm{e}-02+$ & $8.058 \mathrm{e}-01 / 2.346 \mathrm{e}-02+$ \\
\hline
\end{tabular}

Table 5 Mean and standard deviation results of IGD obtained by Tk-MaOEA, MOEA/D, MOMBII, VaEA and NSGA-III on WFGs 1-9 (The best items are in bold).

\begin{tabular}{|c|c|c|c|c|c|c|}
\hline Problem & M & Tk-MaOEA & MOEA/D & MOMBII & VaEA & NSGA-III \\
\hline \multirow{4}{*}{ WFG1 } & 3 & $1.760 \mathrm{e}-01 / 5.277 \mathrm{e}-03$ & $3.080 \mathrm{e}-01 / 3.297 \mathrm{e}-03+$ & $1.704 \mathrm{e}-01 / 1.121 \mathrm{e}-02 \approx$ & $1.717 \mathrm{e}-01 / 1.515 \mathrm{e}-03 \approx$ & $1.460 \mathrm{e}-01 / 3.706 \mathrm{e}-03-$ \\
\hline & 5 & $5.407 \mathrm{e}-01 / 4.968 \mathrm{e}-02$ & $1.232 \mathrm{e}+00 / 6.811 \mathrm{e}-03+$ & $6.556 \mathrm{e}-01 / 1.699 \mathrm{e}-02+$ & $5.231 \mathrm{e}-01 / 3.733 \mathrm{e}-02-$ & $4.981 \mathrm{e}-01 / 1.562 \mathrm{e}-02-$ \\
\hline & 8 & $1.127 e-01 / 8.786 e-02$ & $2.092 \mathrm{e}+00 / 5.591 \mathrm{e}-02+$ & $2.280 \mathrm{e}+00 / 8.718 \mathrm{e}-01+$ & $1.082 \mathrm{e}+00 / 1.861 \mathrm{e}-02+$ & $1.060 \mathrm{e}+00 / 7.159 \mathrm{e}-02+$ \\
\hline & 10 & $2.174 \mathrm{e}-01 / 3.023 \mathrm{e}-02$ & $2.402 \mathrm{e}+00 / 1.310 \mathrm{e}-01+$ & $3.154 \mathrm{e}+00 / 6.767 \mathrm{e}-02+$ & $1.437 \mathrm{e}+00 / 4.106 \mathrm{e}-03-$ & $1.973 \mathrm{e}+00 / 1.257 \mathrm{e}-01-$ \\
\hline \multirow{4}{*}{ WFG2 } & 3 & $1.350 \mathrm{e}-01 / 4.138 \mathrm{e}-01$ & $9.901 \mathrm{e}-01 / 6.738 \mathrm{e}-03+$ & $2.626 \mathrm{e}-01 / 3.454 \mathrm{e}-02+$ & $2.327 \mathrm{e}-01 / 9.639 \mathrm{e}-03+$ & $1.862 \mathrm{e}-01 / 2.258 \mathrm{e}-03+$ \\
\hline & 5 & $2.032 e-01 / 4.317 e-01$ & $5.141 \mathrm{e}+00 / 3.820 \mathrm{e}-02+$ & $1.017 \mathrm{e}+00 / 2.392 \mathrm{e}-01+$ & $8.232 \mathrm{e}-01 / 1.523 \mathrm{e}-02+$ & 7.926e-01/3.736e-02+ \\
\hline & 8 & $7.341 \mathrm{e}+00 / 2.133 \mathrm{e}+00$ & $8.706 \mathrm{e}+00 / 4.979 \mathrm{e}-02+$ & $1.698 \mathrm{e}+00 / 2.264 \mathrm{e}-02-$ & $2.728 \mathrm{e}+00 / 8.891 \mathrm{e}-02-$ & $4.553 \mathrm{e}+00 / 5.976 \mathrm{e}-01-$ \\
\hline & 10 & $1.169 e+00 / 7.871 e+00$ & $1.647 \mathrm{e}+01 / 8.062 \mathrm{e}-02+$ & $2.569 \mathrm{e}+00 / 2.132 \mathrm{e}-02+$ & $2.823 \mathrm{e}+00 / 7.782 \mathrm{e}-02+$ & $5.943 \mathrm{e}+00 / 8.279 \mathrm{e}-02+$ \\
\hline \multirow{4}{*}{ WFG3 } & 3 & $2.143 \mathrm{e}-01 / 8.795 \mathrm{e}-02$ & $1.581 \mathrm{e}-01 / 8.019 \mathrm{e}-04-$ & 9.327e-02/5.548e-03- & $1.374 \mathrm{e}-01 / 2.980 \mathrm{e}-02-$ & $1.042 \mathrm{e}-01 / 4.523 \mathrm{e}-03-$ \\
\hline & 5 & $4.861 \mathrm{e}-01 / 2.223 \mathrm{e}-01$ & $8.676 \mathrm{e}-01 / 7.252 \mathrm{e}-03+$ & $6.518 \mathrm{e}-01 / 2.447 \mathrm{e}-01+$ & $6.412 \mathrm{e}-01 / 5.700 \mathrm{e}-02+$ & $6.102 \mathrm{e}-01 / 8.503 \mathrm{e}-02+$ \\
\hline & 8 & $8.740 \mathrm{e}+00 / 2.059 \mathrm{e}-02$ & $3.808 \mathrm{e}+00 / 5.686 \mathrm{e}-02-$ & $8.648 \mathrm{e}+00 / 8.009 \mathrm{e}-04-$ & $1.711 \mathrm{e}+00 / 8.502 \mathrm{e}-02-$ & $1.363 \mathrm{e}+00 / 7.676 \mathrm{e}-01-$ \\
\hline & 10 & $9.731 \mathrm{e}+00 / 2.807 \mathrm{e}-02$ & $5.701 \mathrm{e}+00 / 6.046 \mathrm{e}-02-$ & $1.093 \mathrm{e}+01 / 2.134 \mathrm{e}-03+$ & $2.321 \mathrm{e}+00 / 5.243 \mathrm{e}-02-$ & $2.696 \mathrm{e}+00 / 1.418 \mathrm{e}-01-$ \\
\hline WFG4 & 3 & $1.894 \mathrm{e}-01 / 2.149 \mathrm{e}-01$ & $2.423 \mathrm{e}-01 / 5.531 \mathrm{e}-04+$ & $2.214 \mathrm{e}-01 / 1.893 \mathrm{e}-04+$ & $2.207 \mathrm{e}-01 / 7.216 \mathrm{e}-03+$ & $2.210 \mathrm{e}-01 / 1.228 \mathrm{e}-04+$ \\
\hline
\end{tabular}




\begin{tabular}{|c|c|c|c|c|c|c|}
\hline & 5 & $3.331 \mathrm{e}+00 / 5.437 \mathrm{e}-01$ & $1.821 \mathrm{e}+00 / 4.664 \mathrm{e}-02-$ & $1.316 \mathrm{e}+00 / 1.248 \mathrm{e}-02-$ & $1.187 e+00 / 9.580 e-03-$ & $1.226 \mathrm{e}+00 / 5.037 \mathrm{e}-04-$ \\
\hline & 8 & $6.011 \mathrm{e}+00 / 1.686 \mathrm{e}-01$ & $7.126 \mathrm{e}+00 / 3.023 \mathrm{e}-03+$ & $5.082 \mathrm{e}+00 / 1.916 \mathrm{e}+00-$ & $3.375 \mathrm{e}+00 / 1.257 \mathrm{e}-01-$ & $3.547 \mathrm{e}+00 / 1.260 \mathrm{e}-02-$ \\
\hline & 10 & $4.793 \mathrm{e}+00 / 2.823 \mathrm{e}-01$ & $9.599 \mathrm{e}+00 / 2.649 \mathrm{e}-01+$ & $1.125 \mathrm{e}+01 / 1.575 \mathrm{e}+00+$ & $5.013 \mathrm{e}+00 / 6.821 \mathrm{e}-02+$ & $6.066 \mathrm{e}+00 / 2.834 \mathrm{e}-01+$ \\
\hline \multirow{4}{*}{ WFG5 } & 3 & $2.458 \mathrm{e}-01 / 4.052 \mathrm{e}-01$ & $2.461 \mathrm{e}-01 / 1.470 \mathrm{e}-03 \approx$ & $2.402 \mathrm{e}-01 / 2.681 \mathrm{e}-03 \approx$ & $2.325 \mathrm{e}-01 / 4.141 \mathrm{e}-03-$ & $2.298 \mathrm{e}-01 / 4.884 \mathrm{e}-05-$ \\
\hline & 5 & $4.009 \mathrm{e}+00 / 3.617 \mathrm{e}-01$ & $1.672 \mathrm{e}+00 / 6.596 \mathrm{e}-02-$ & $1.319 \mathrm{e}+00 / 1.743 \mathrm{e}-02-$ & $1.172 \mathrm{e}+00 / 1.202 \mathrm{e}-03-$ & $1.215 \mathrm{e}+00 / 3.882 \mathrm{e}-05-$ \\
\hline & 8 & $3.142 \mathrm{e}+00 / 9.949 \mathrm{e}-01$ & $6.901 \mathrm{e}+00 / 7.500 \mathrm{e}-02+$ & $7.348 \mathrm{e}+00 / 5.070 \mathrm{e}+00+$ & $3.404 \mathrm{e}+00 / 2.216 \mathrm{e}-02+$ & $3.522 \mathrm{e}+00 / 6.983 \mathrm{e}-03+$ \\
\hline & 10 & $4.524 \mathrm{e}+00 / 8.001 \mathrm{e}-01$ & $9.409 \mathrm{e}+00 / 1.910 \mathrm{e}-01+$ & $1.473 \mathrm{e}+01 / 6.683 \mathrm{e}-01+$ & $5.029 \mathrm{e}+00 / 7.102 \mathrm{e}-02+$ & $5.827 \mathrm{e}+00 / 1.797 \mathrm{e}-02+$ \\
\hline \multirow{4}{*}{ WFG6 } & 3 & $3.392 \mathrm{e}-01 / 8.254 \mathrm{e}-01$ & $2.791 \mathrm{e}-01 / 1.516 \mathrm{e}-02-$ & $2.557 \mathrm{e}-01 / 1.600 \mathrm{e}-02-$ & $2.507 \mathrm{e}-01 / 9.128 \mathrm{e}-03-$ & $2.339 \mathrm{e}-01 / 1.075 \mathrm{e}-02-$ \\
\hline & 5 & $5.393 \mathrm{e}+00 / 2.229 \mathrm{e}-01$ & $2.007 \mathrm{e}+00 / 1.464 \mathrm{e}-01-$ & $1.522 \mathrm{e}+00 / 6.483 \mathrm{e}-02-$ & $1.192 e+00 / 8.777 e-04-$ & $1.214 \mathrm{e}+00 / 3.967 \mathrm{e}-04-$ \\
\hline & 8 & $2.870 \mathrm{e}+00 / 5.296 \mathrm{e}-01$ & $7.474 \mathrm{e}+00 / 8.822 \mathrm{e}-02+$ & $3.676 \mathrm{e}+00 / 1.123 \mathrm{e}-01+$ & $3.467 \mathrm{e}+00 / 4.930 \mathrm{e}-02+$ & $3.989 \mathrm{e}+00 / 6.283 \mathrm{e}-01+$ \\
\hline & 10 & $1.078 \mathrm{e}+01 / 1.165 \mathrm{e}-02$ & $1.068 \mathrm{e}+01 / 7.492 \mathrm{e}-02-$ & $6.547 \mathrm{e}+00 / 8.831 \mathrm{e}-01-$ & $5.062 \mathrm{e}+00 / 1.513 e-02-$ & $5.874 \mathrm{e}+00 / 3.739 \mathrm{e}-02-$ \\
\hline \multirow{4}{*}{ WFG7 } & 3 & $3.432 \mathrm{e}-01 / 4.365 \mathrm{e}-01$ & $2.574 \mathrm{e}-01 / 3.499 \mathrm{e}-03-$ & $2.210 \mathrm{e}-01 / 1.542 \mathrm{e}-04-$ & $2.218 \mathrm{e}-01 / 8.593 \mathrm{e}-03-$ & $2.211 \mathrm{e}-01 / 3.630 \mathrm{e}-05-$ \\
\hline & 5 & $5.350 \mathrm{e}+00 / 4.979 \mathrm{e}-01$ & $1.958 \mathrm{e}+00 / 1.410 \mathrm{e}-01-$ & $1.238 \mathrm{e}+00 / 7.903 \mathrm{e}-03-$ & $1.184 e+00 / 1.038 e-02-$ & $1.229 \mathrm{e}+00 / 1.192 \mathrm{e}-03-$ \\
\hline & 8 & $3.013 \mathrm{e}+00 / 4.567 \mathrm{e}-01$ & $7.436 \mathrm{e}+00 / 2.039 \mathrm{e}-01+$ & $3.652 \mathrm{e}+00 / 8.816 \mathrm{e}-02+$ & $3.406 \mathrm{e}+00 / 3.566 \mathrm{e}-02+$ & $4.098 \mathrm{e}+00 / 7.650 \mathrm{e}-01+$ \\
\hline & 10 & $4.300 \mathrm{e}+00 / 3.529 \mathrm{e}-01$ & $1.025 \mathrm{e}+01 / 5.878 \mathrm{e}-01+$ & $7.881 \mathrm{e}+00 / 2.916 \mathrm{e}-01+$ & $4.976 \mathrm{e}+00 / 6.704 \mathrm{e}-02+$ & $5.950 \mathrm{e}+00 / 6.381 \mathrm{e}-02+$ \\
\hline \multirow{4}{*}{ WFG8 } & 3 & $3.544 \mathrm{e}-01 / 2.724 \mathrm{e}-01$ & $2.916 \mathrm{e}-01 / 3.475 \mathrm{e}-04-$ & $3.212 \mathrm{e}-01 / 9.175 \mathrm{e}-04-$ & $2.972 \mathrm{e}-01 / 5.659 \mathrm{e}-03-$ & 2.797e-01/2.997e-03- \\
\hline & 5 & $5.569 \mathrm{e}+00 / 9.027 \mathrm{e}-02$ & $1.940 \mathrm{e}+00 / 2.267 \mathrm{e}-02-$ & $1.867 \mathrm{e}+00 / 1.029 \mathrm{e}-02-$ & $1.256 \mathrm{e}+00 / 4.158 \mathrm{e}-03-$ & $1.232 \mathrm{e}+00 / 1.371 \mathrm{e}-03-$ \\
\hline & 8 & $8.104 \mathrm{e}+00 / 6.591 \mathrm{e}-01$ & $6.712 \mathrm{e}+00 / 3.112 \mathrm{e}-01-$ & $6.787 \mathrm{e}+00 / 4.186 \mathrm{e}-01-$ & $3.504 \mathrm{e}+00 / 8.002 \mathrm{e}-03-$ & $4.452 \mathrm{e}+00 / 2.208 \mathrm{e}-02-$ \\
\hline & 10 & $4.775 e+00 / 7.688 e-01$ & $6.553 \mathrm{e}+00 / 4.670 \mathrm{e}-01+$ & $1.010 \mathrm{e}+01 / 8.070 \mathrm{e}-01+$ & $5.204 \mathrm{e}+00 / 2.600 \mathrm{e}-02+$ & $6.263 \mathrm{e}+00 / 3.764 \mathrm{e}-01+$ \\
\hline \multirow{4}{*}{ WFG9 } & 3 & $1.093 \mathrm{e}-01 / 1.751 \mathrm{e}-01$ & $2.423 \mathrm{e}-01 / 5.682 \mathrm{e}-03-$ & $2.647 \mathrm{e}-01 / 1.665 \mathrm{e}-03-$ & $2.208 \mathrm{e}-01 / 1.041 \mathrm{e}-03-$ & $2.211 \mathrm{e}-01 / 1.854 \mathrm{e}-04-$ \\
\hline & 5 & $3.070 \mathrm{e}+00 / 2.262 \mathrm{e}-01$ & $1.790 \mathrm{e}+00 / 2.385 \mathrm{e}-01-$ & $1.771 \mathrm{e}+00 / 2.086 \mathrm{e}-02-$ & $1.150 \mathrm{e}+00 / 1.491 \mathrm{e}-04-$ & $1.213 \mathrm{e}+00 / 1.639 \mathrm{e}-02-$ \\
\hline & 8 & $3.245 \mathrm{e}+00 / 5.305 \mathrm{e}-01$ & $6.970 \mathrm{e}+00 / 7.205 \mathrm{e}-02+$ & $3.738 \mathrm{e}+00 / 2.064 \mathrm{e}-02+$ & $3.310 \mathrm{e}+00 / 5.061 \mathrm{e}-02+$ & $3.570 \mathrm{e}+00 / 1.802 \mathrm{e}-02+$ \\
\hline & 10 & $7.816 \mathrm{e}+00 / 1.944 \mathrm{e}+00$ & $1.017 \mathrm{e}+01 / 3.305 \mathrm{e}-01+$ & $1.700 \mathrm{e}+01 / 1.597 \mathrm{e}-01+$ & $4.916 \mathrm{e}+00 / 2.970 \mathrm{e}-02-$ & $5.681 \mathrm{e}+00 / 2.369 \mathrm{e}-01-$ \\
\hline
\end{tabular}




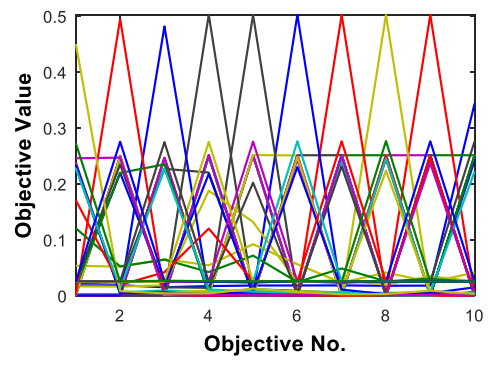

(a) Tk-MaOEA

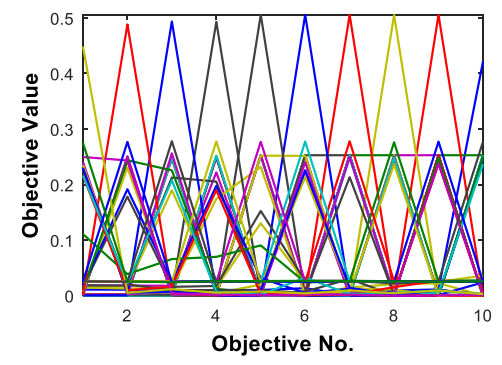

(b) MOEA/D

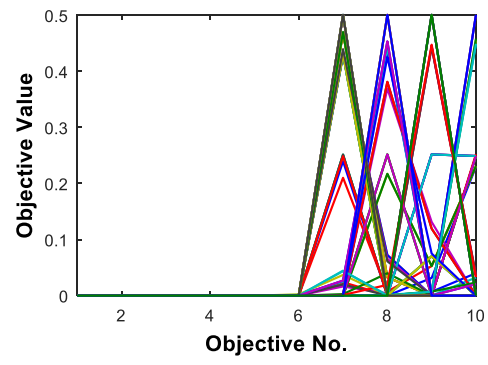

(c) MOMBII

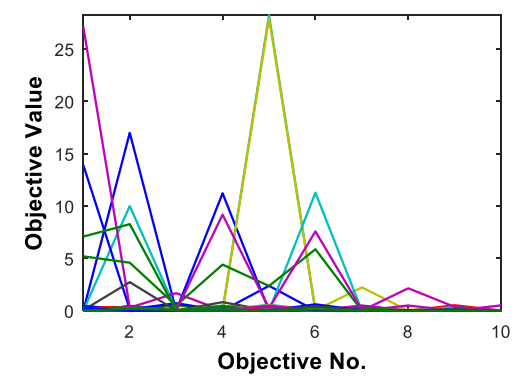

(d) VaEA

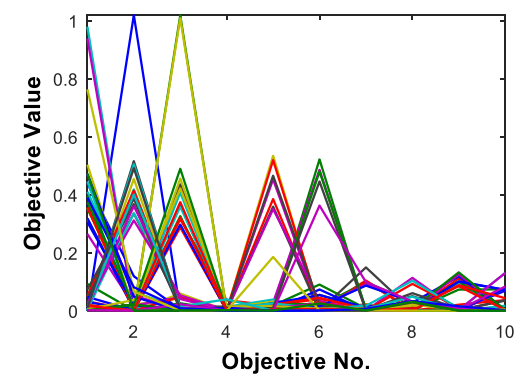

(e) NSGA-III

Fig.3. Final solution set of involved algorithms on the 10-objective DTLZ1, shown by parallel coordinates

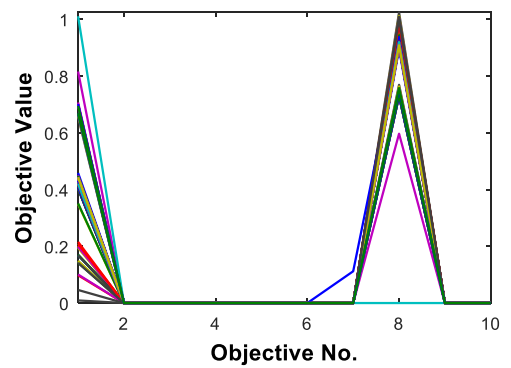

(a) Tk-MaOEA

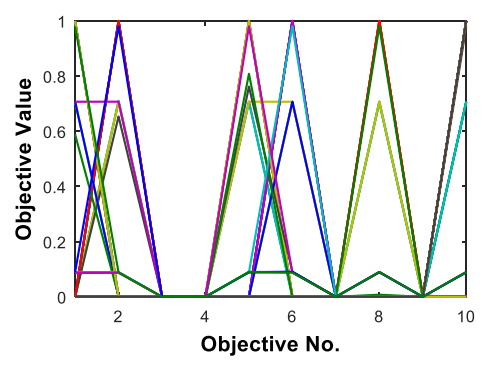

(b) MOEA/D

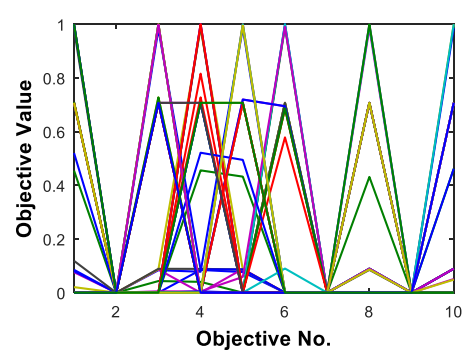

(c) MOMBII

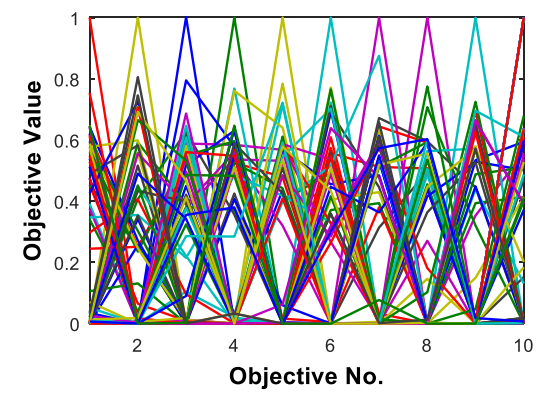

(d) VaEA

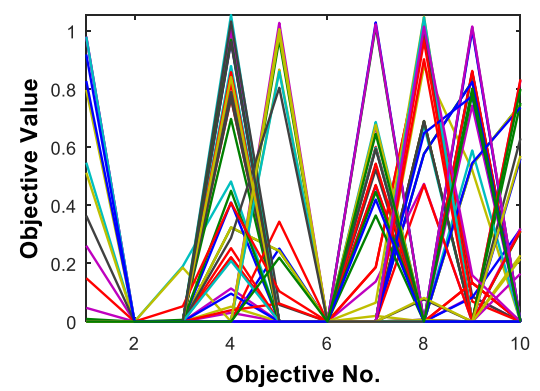

(e) NSGA-III

Fig.4. Final solution set of involved algorithms on the 10-objective DTLZ4, shown by parallel coordinates 


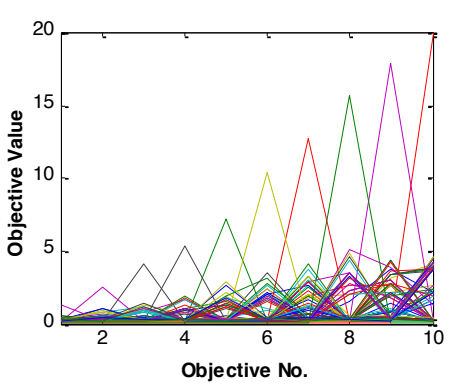

(a) Tk-MaOEA

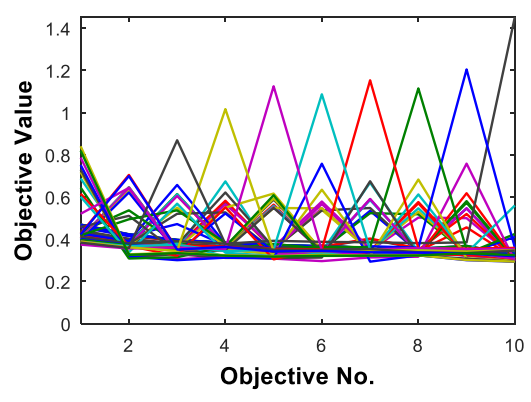

(b) MOEA/D

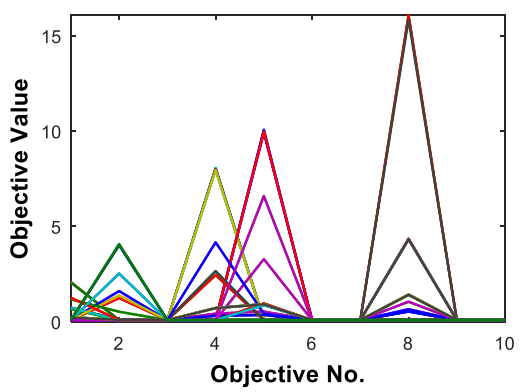

(c) MOMBII

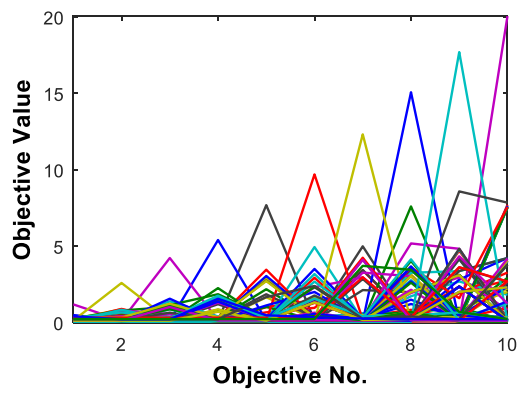

(d) VaEA

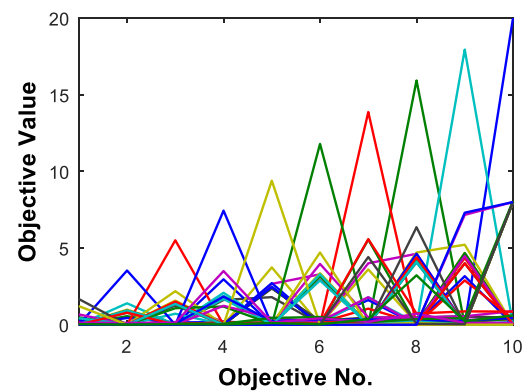

(e) NSGA-III

Fig.5. Final solution set of involved algorithms on the 10-objective WFG1, shown by parallel coordinates

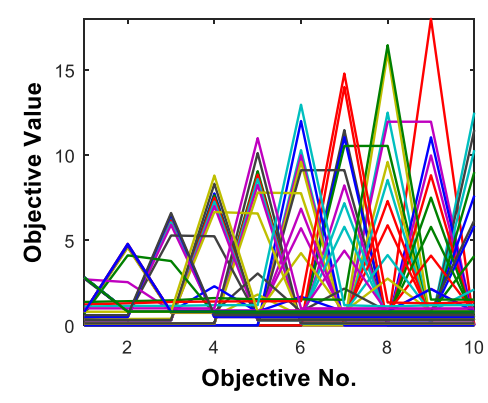

(a) Tk-MaOEA

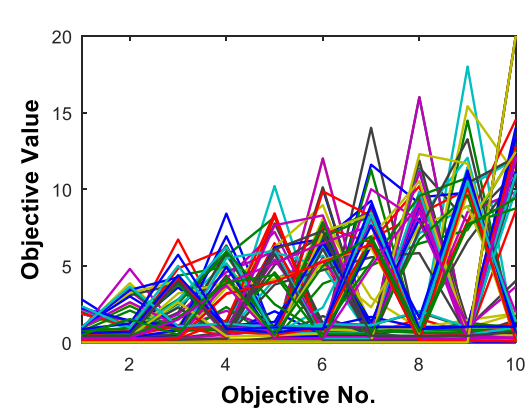

(d) VaEA

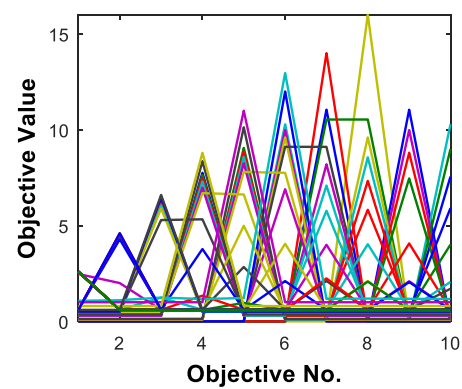

(b) $M O E A / D$

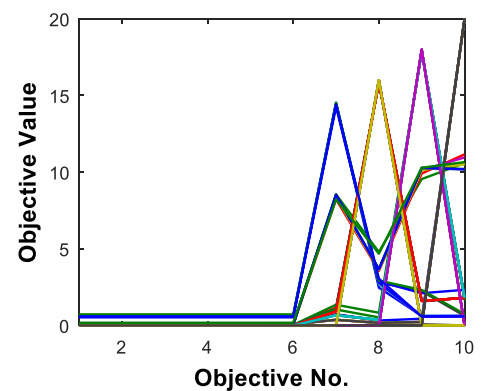

(c) MOMBII
Fig.6. Final solution set of involved algorithms on the 10-objective WFG8, shown by parallel coordinates 


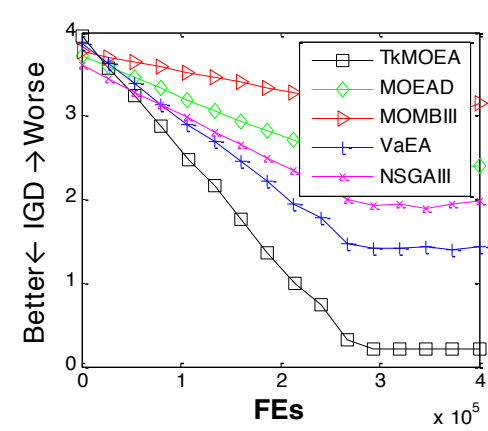

(a) WFG1

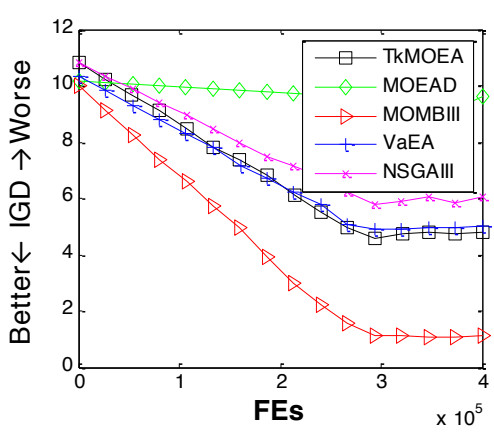

(d) WFG4

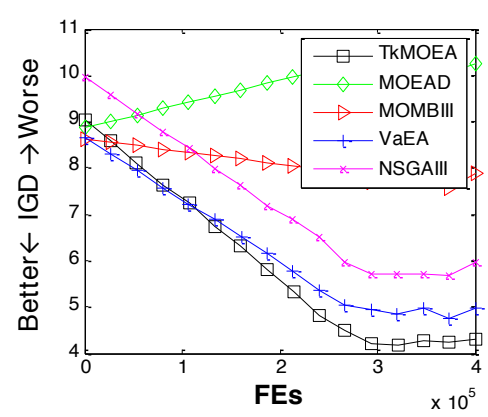

(g) WFG7

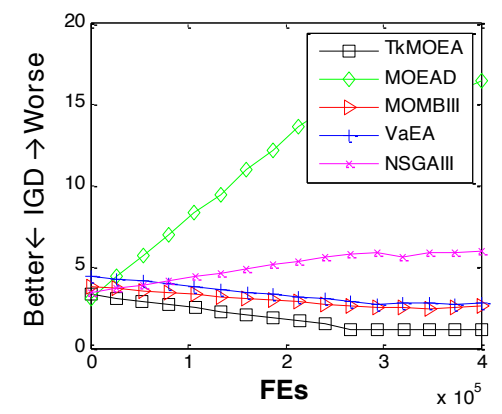

(b) WFG2

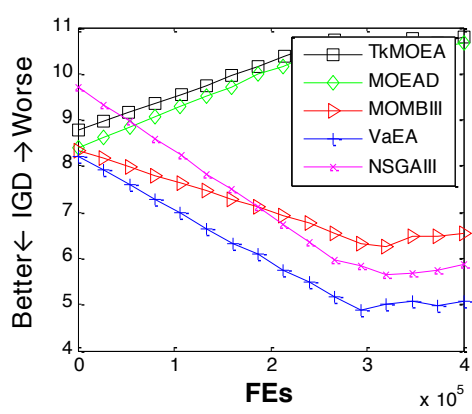

(e) WFG5

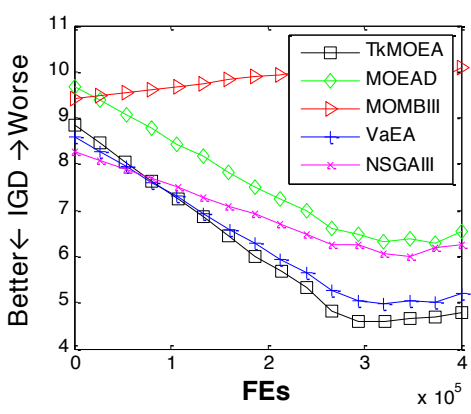

(h) WFG8

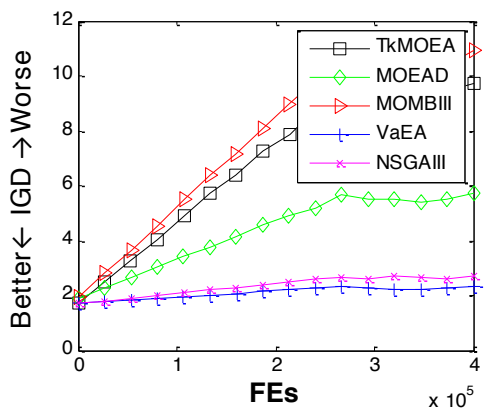

(c) WFG3

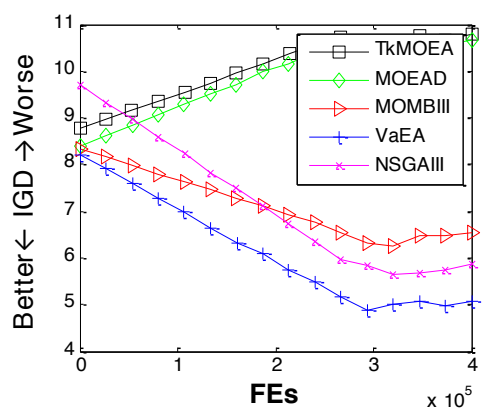

(f) WFG6

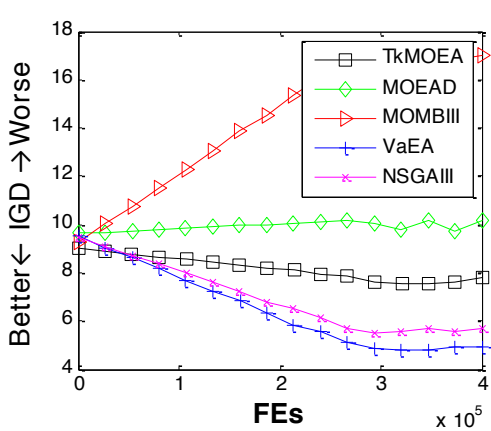

(i) WFG9

Fig.7. Evolutionary trajectories of IGD on all the 10-objective WFG test problems

\subsubsection{Results in terms of IGD metric}

Comparative results in terms of IGD values on WFGs obtained by each algorithm are given in Table 5. It can be observed from this table that Tk-MaOEA obtains the first ranks on 15 of the 36 test instances, while NSGA-III does best on 7 of the 36 instances. For VaEA, it also performs best on 10 test instances, including 10-objective WFG1, 10-objective WFG3, 5- and 8-objective WFG4, 5-objective WFG5, 5- and 10-objective WFG6, 5-objecitve WFG7, 8-objective WFG8 and 5-objective WFG9. MOMBII and MOEA/D obtains satisfactory results on 5-objective WFG2, 3-objective WFG3, 3-objective WFG7 and 10-objective WFG9. It is stressed that for test instances with complicated PFs such as WFG1 and WFG2, and with larger number of 
objectives such as 10-objective WFG7 and WFG8, the performance of Tk-MaOEA is more competent than that of other algorithms.

Figs. 3-6 respectively give the plot of final solution set obtained by involved algorithms on the 10-objective DTLZ1, DTLZ4, WFG1 and WFG8, shown by parallel coordinates. From these figures, we can see final solutions found by Tk-MaOEA are superior in terms of both convergence and distribution to other algorithms. For 10-objective WFG3, it can observed from Fig. 5 that the distribution of solutions obtained by Tk-MaOEA exhibits excellent convergence and diversity, while other algorithms do relatively poor. Especially, for 10-objective WFG8, we can see from Fig.6 that NSGA-III also achieves satisfactory performance, but still slightly concentres on the middle part of several objectives. This example also indicates that Tk-MaOEA is capable of working effectively on difficult multimodal problems.

Fig.7 shows the plot of evolutionary process of involved algorithm in terms of IGD values versus the number of generations. From the figure, it can be observed that Tk-MaOEA has a faster convergence speed than other algorithms and finds the best IGD value on most of test instances, which shows evidence to support the discussion of the proposed scheme in Section III-I. To be specific, On WFG1, the convergence of Tk-MaOEA is obviously superior to other algorithms, and MOMBII performs the worst. On WFG2, Tk-MaOEA and VaEA exhibits promising performance. For WFG3, WFG5 and WFG6, Tk-MaOEA performs a little worse than other algorithms. However, for WFG4, WFG7-WFG9, Tk-MaOEA performs better or at least comparably with NSGA-III, better than other algorithms, which experimentally verifies the efficiency of the proposed scheme in Tk-MaOEA.

\subsubsection{Statistical result analysis}

Performance comparisons between algorithms are conducted based on a rigorous nonparametric statistical method, i.e., Friedman test [71], which is used to detect differences in treatments across multiple test attempts. For each benchmark instance, the performance metric values (IGD and HV) of each algorithm are calculated as shown in Tables 4 and 5. Then the statistical test is used with the performance metric values. We first test the hypothesis that all algorithms perform equally using the Friedman test. If this hypothesis is rejected at the $95 \%$ confidence level, we then consider pair-wise comparisons between the algorithms using the Wilcoxon's s rank sum test at the $95 \%$ confidence level.

Tables 6 and 7 show the results of the Friedman test, where $p$ is the probability for the chi-square statistic, to determine whether the hypothesis is rejected (if $p<0.05$ ), and the meanings of other parameters such as SS (sum of squares), df (degrees of freedom), MS (the ratio SS/df), columns and errors can be referred to Friedman 
function declarations in Matlab. From these tables, the initial Friedman test breaks the hypothesis that all five algorithms are equivalent. Therefore, the outcomes of pair-wise statistical comparisons for IGD and HV are shown in Tables 8 and 9, respectively. Here R+ represents the sum of ranks for the test instances on which the first algorithm outperforms the latter one, and R- means the sum of ranks for the opposite. As shown in Table 8, we can that that all p-values are less than 0.05 , which strongly indicates that the performance of Tk-MaOEA is statistically superior to other algorithms on the DTLZ and WFG test instances in terms of IGD metric. Likewise, computation results in Table 9 with aspect to the HV metric, show that the performance of Tk-MaOEA on these WFG instances is statistically more powerful than the compared algorithms.

Table 6. Statistical results by Friedman ranking for HV-metric considering DTLZs and WFGs

\begin{tabular}{|c|c|c|c|c|c|}
\hline Source & SS & $\mathrm{df}$ & MS & Chi-sq & $\mathrm{p}$ \\
\hline Columns & 113.635 & 4 & 28.4087 & 45.72 & 2.81967E-09 \\
\hline Error & 403.365 & 204 & 1.9773 & & \\
\hline \multirow[t]{2}{*}{ Total } & 517 & 259 & & & \\
\hline & \multicolumn{5}{|c|}{ Table 7. Statistical results by Friedman ranking for IGD-metric considering WFGs } \\
\hline Source & SS & $\mathrm{df}$ & MS & Chi-sq & $\mathrm{p}$ \\
\hline Columns & 144.5 & 4 & 36.375 & 57.67 & $8.9648 \mathrm{E}-12$ \\
\hline Error & 215.5 & 140 & 1.5893 & & \\
\hline Total & 360 & 179 & & & \\
\hline
\end{tabular}

Table 8. Statistical results by Wilcoxon test for HV-metric considering DTLZs and WFGs

\begin{tabular}{lccc}
\hline Tk-MaOEA vs. & R+ & R- & p-value \\
\hline MOEA/D & 46 & 3 & $5.634 \mathrm{E}-4$ \\
MOMBII & 35 & 13 & $1.534 \mathrm{E}-3$ \\
VaEA & 36 & 9 & $1.782 \mathrm{E}-3$ \\
NSGA-III & 28 & 14 & $4.034 \mathrm{E}-3$
\end{tabular}

Table 9. Statistical results by Wilcoxon test for IGD-metric considering WFGs

\begin{tabular}{lccc}
\hline Tk-MaOEA vs. & $\mathrm{R}+$ & $\mathrm{R}-$ & $\mathrm{p}$-value \\
\hline MOEA/D & 30 & 6 & $1.034 \mathrm{E}-3$ \\
MOMBII & 28 & 8 & $4.374 \mathrm{E}-3$ \\
VaEA & 24 & 12 & $6.723 \mathrm{E}-3$ \\
NSGA-III & 20 & 16 & $1.116 \mathrm{E}-2$ \\
\hline
\end{tabular}




\subsection{Further Analysis}

\subsubsection{Effect of transfer learning and Kriging model}

In order to further investigate the effectiveness of algorithm components, three variants, namely Tk-MOEA, Tk-MOEA-TL (only with transfer learning), and Tk-MOEA-K (only with Kriging model) are tested on the DTLZ and WFG problems with 10 objectives. Table 10 shows the results of the three variants, regarding the mean and SD values in terms of IGD metric, where the better results are highlighted in bold.

As can be seen from Table 10, when transfer learning is incorporated into the algorithm, the performance of Tk-MOEA-TL has a clear improvement over that of Tk-MOEA-K, achieving a better value on 3-, 5-, and 8-objective DTLZ1, 8-objective DTLZ3, and 3-objective WFG4 while Tk-MOEA-K performs better than Tk-MOEA-TL on 10-objective DTLZ1, 3-objective DTLZ2 and 8-objective WFG4. The PF shape of DTLZ3 is composed of a set of discontinuous segments, and the distribution of the points on PF of DTLZ4 is strongly nonuniform.

Generally, these two benchmarks are more difficult to be tackled compared with DTLZ1 and DTLZ2. This implies that both transfer learning and Kriging model can affect the performance of the algorithm, while transfer learning may be more effective in complex test functions. For most of the problems, Tk-MOEA shows an advantage over its competitors, which means that the proposed two mechanisms can work together effectively.

\subsubsection{Incorporating transfer learning into NSGA-III}

In this section, we apply transfer learning to the classical NSGA-III, namely Tk-NSGA-III. NSGA-III is known for its nondominated sorting and reference-based guidance strategies. The improved algorithm is compared with its original version and a Kriging-based algorithm called GeDEA-II-K [57]. The GeDEA-II is a multi-objective real-coded evolutionary algorithm based on a Pareto-like evaluation method [72]. GeDEA-II-K is to improve the GeDEA-II's reproduction operator with the integration of a Kriging filter [73]. Table 11 shows the comparative results of the three algorithms on the DTLZ and WFG problems with 10 objectives. Tk-NSGA-III outperforms the original NSGA-III on most of the selected benchmarks. Tk-NSGA-III achieves a better value on 10-objective DTLZ1, DTLZ2, DTLZ3, DTLZ4, WFG1, WFG2 and WFG8, and with better SD values on 11 test instances. NSGA-III obtains a better value on the majority of DTLZ1, DTLZ2 and WFG9. In addition, GeDEA-II-K also obtains a satisfactory result on WFG2 and WFG4. From these results, it can be observed that the transfer learning approach can play a positive effect on the performance of NSGA-III. 
Table 10 Mean and standard deviation results of IGD obtained by Tk-MaOEA, Tk-MaOEA-TL, Tk-MaOEA-K

\begin{tabular}{|c|c|c|c|c|}
\hline Problem & M & Tk-MaOEA & Tk-MaOEA-TL & MaOEA-K \\
\hline \multirow{4}{*}{ DTLZ1 } & 3 & $1.634 \mathrm{e}-02 / 9.257 \mathrm{e}-03$ & $1.517 \mathrm{e}-02 / 2.870 \mathrm{e}-02-$ & $1.714 \mathrm{e}-02 / 1.928 \mathrm{e}-01+$ \\
\hline & 5 & $1.682 \mathrm{e}-02 / 7.574 \mathrm{e}-03$ & $1.621 \mathrm{e}-02 / 9.298 \mathrm{e}-03-$ & $9.329 \mathrm{e}-02 / 4.658 \mathrm{e}-01+$ \\
\hline & 8 & $1.851 \mathrm{e}-01 / 1.077 \mathrm{e}-03$ & 1.794e-01/3.932e-03- & $1.919 \mathrm{e}-01 / 4.018 \mathrm{e}-03+$ \\
\hline & 10 & $1.795 \mathrm{e}-01 / 2.166 \mathrm{e}-03$ & $1.707 \mathrm{e}-01 / 1.987 \mathrm{e}-04-$ & $1.547 \mathrm{e}-01 / 1.283 \mathrm{e}-03-$ \\
\hline \multirow{4}{*}{ DTLZ2 } & 3 & $6.371 \mathrm{e}-02 / 3.864 \mathrm{e}-02$ & $4.720 \mathrm{e}-02 / 6.150 \mathrm{e}-02-$ & $1.324 \mathrm{e}-02 / 4.311 \mathrm{e}-03-$ \\
\hline & 5 & $1.838 \mathrm{e}-01 / 1.781 \mathrm{e}-01$ & $5.869 \mathrm{e}-01 / 8.540 \mathrm{e}-02+$ & $2.435 \mathrm{e}-01 / 3.284 \mathrm{e}-02+$ \\
\hline & 8 & $2.131 \mathrm{e}-01 / 2.210 \mathrm{e}-01$ & $7.211 \mathrm{e}-01 / 2.503 \mathrm{e}-02+$ & $2.552 \mathrm{e}-01 / 2.264 \mathrm{e}-02+$ \\
\hline & 10 & $1.083 \mathrm{e}-01 / 3.899 \mathrm{e}-02$ & $8.313 \mathrm{e}-01 / 5.503 \mathrm{e}-02+$ & $2.619 \mathrm{e}-01 / 7.383 \mathrm{e}-03+$ \\
\hline \multirow{4}{*}{ DTLZ3 } & 3 & $1.076 \mathrm{e}-02 / 6.160 \mathrm{e}-01$ & $3.809 \mathrm{e}-02 / 2.546 \mathrm{e}-02+$ & $7.633 \mathrm{e}-02 / 3.629 \mathrm{e}-02+$ \\
\hline & 5 & $9.215 \mathrm{e}-01 / 2.894 \mathrm{e}-02$ & $7.025 \mathrm{e}-01 / 1.213 \mathrm{e}-01-$ & 4.971e-01/8.148e-01- \\
\hline & 8 & $9.365 \mathrm{e}-01 / 1.251 \mathrm{e}-02$ & 7.103e-01/8.305e-03- & $1.487 \mathrm{e}+00 / 2.113 \mathrm{e}-02+$ \\
\hline & 10 & $9.425 \mathrm{e}-01 / 1.734 \mathrm{e}-01$ & $8.413 \mathrm{e}-01 / 1.060 \mathrm{e}-01-$ & $1.551 \mathrm{e}+00 / 1.060 \mathrm{e}-02+$ \\
\hline \multirow{4}{*}{ DTLZ4 } & 3 & $5.665 \mathrm{e}-01 / 1.727 \mathrm{e}-02$ & $9.459 \mathrm{e}-01 / 9.557 \mathrm{e}-10+$ & $3.336 \mathrm{e}-01 / 2.960 \mathrm{e}-01-$ \\
\hline & 5 & $8.686 \mathrm{e}-01 / 6.550 \mathrm{e}-04$ & $9.890 \mathrm{e}-01 / 1.684 \mathrm{e}-01+$ & 2.435e-01/1.051e-01- \\
\hline & 8 & $1.057 \mathrm{e}-01 / 5.477 \mathrm{e}-03$ & $8.931 \mathrm{e}-01 / 2.045 \mathrm{e}-01+$ & $2.600 \mathrm{e}-01 / 2.303 \mathrm{e}-02+$ \\
\hline & 10 & $1.197 \mathrm{e}-01 / 1.970 \mathrm{e}-02$ & $9.157 \mathrm{e}-01 / 7.183 \mathrm{e}-02+$ & $2.647 \mathrm{e}-01 / 1.057 \mathrm{e}-02+$ \\
\hline \multirow{4}{*}{ WFG1 } & 3 & $1.760 \mathrm{e}-01 / 5.277 \mathrm{e}-03$ & $1.994 \mathrm{e}-01 / 1.014 \mathrm{e}-02+$ & $1.832 \mathrm{e}-01 / 2.929 \mathrm{e}-01+$ \\
\hline & 5 & $5.407 \mathrm{e}-01 / 4.968 \mathrm{e}-02$ & $2.297 \mathrm{e}-01 / 8.330 \mathrm{e}-03-$ & $1.316 e-01 / 1.535 e-01-$ \\
\hline & 8 & $1.127 \mathrm{e}-01 / 8.786 \mathrm{e}-02$ & $2.932 \mathrm{e}-01 / 2.824 \mathrm{e}-03+$ & $1.697 \mathrm{e}-01 / 1.503 \mathrm{e}-01+$ \\
\hline & 10 & $2.174 \mathrm{e}-01 / 3.023 \mathrm{e}-02$ & $3.067 \mathrm{e}-01 / 2.563 \mathrm{e}-02+$ & $2.201 \mathrm{e}-01 / 9.423 \mathrm{e}-02+$ \\
\hline \multirow{4}{*}{ WFG4 } & 3 & $1.894 \mathrm{e}-01 / 2.149 \mathrm{e}-01$ & $1.619 \mathrm{e}-01 / 1.150 \mathrm{e}-01-$ & $3.552 \mathrm{e}-01 / 1.829 \mathrm{e}-02+$ \\
\hline & 5 & $3.331 \mathrm{e}+00 / 5.437 \mathrm{e}-01$ & $3.747 \mathrm{e}+00 / 4.016 \mathrm{e}-01+$ & $1.396 \mathrm{e}+00 / 7.857 \mathrm{e}-03-$ \\
\hline & 8 & $6.011 \mathrm{e}+00 / 1.686 \mathrm{e}-01$ & $6.214 \mathrm{e}+00 / 4.963 \mathrm{e}-03+$ & $3.415 \mathrm{e}+00 / 5.298 \mathrm{e}-02-$ \\
\hline & 10 & $4.793 e+00 / 2.823 e-01$ & $8.406 \mathrm{e}+00 / 4.290 \mathrm{e}-01+$ & $5.093 \mathrm{e}+00 / 7.764 \mathrm{e}-02+$ \\
\hline
\end{tabular}

\subsubsection{Comparison results with surrogates-based algorithm}

In this section, the proposed algorithm is experimentally compared with an art-of-the-state surrogates-based algorithm, called K-RVEA [74]. In K-RVEA, the Kriging models are used to approximate each objective function to reduce the computational cost. In addition, based on a set of adaptive reference vectors for selection, the convergence and diversity in K-RVEA can be balanced by using the uncertainty information provided by the Kriging models. 
Table 11 Mean and standard deviation results of IGD obtained by Tk-MaOEA (i.e., Tk-NSGA-III), GeDEA-II-K, and NSGA-III

\begin{tabular}{|c|c|c|c|c|}
\hline Problem & M & Tk-MaOEA (Tk-NSGA-III) & GeDEA-II-K & NSGA-III. \\
\hline \multirow{4}{*}{ DTLZ1 } & 3 & $1.634 \mathrm{e}-01 / 9.257 \mathrm{e}-03$ & $1.750 \mathrm{e}-01 / 2.015 \mathrm{e}-01+$ & $2.059 \mathrm{e}-02 / 1.926 \mathrm{e}-05-$ \\
\hline & 5 & $1.682 \mathrm{e}-01 / 7.574 \mathrm{e}-03$ & $1.681 \mathrm{e}+00 / 1.790 \mathrm{e}-01+$ & $6.810 e-02 / 1.085 e-07-$ \\
\hline & 8 & $1.551 \mathrm{e}-01 / 1.077 \mathrm{e}-03$ & $1.315 \mathrm{e}+02 / 8.312 \mathrm{e}+01+$ & $1.584 \mathrm{e}-01 / 4.088 \mathrm{e}-04-$ \\
\hline & 10 & $1.795 \mathrm{e}-01 / 2.166 \mathrm{e}-03$ & $1.624 \mathrm{e}+02 / 4.387 \mathrm{e}+00+$ & $2.451 \mathrm{e}-01 / 2.266 \mathrm{e}-03+$ \\
\hline \multirow{4}{*}{ DTLZ2 } & 3 & $6.371 \mathrm{e}-01 / 3.864 \mathrm{e}-02$ & $1.347 \mathrm{e}-01 / 9.174 \mathrm{e}-03-$ & $5.446 \mathrm{e}-02 / 4.256 \mathrm{e}-07-$ \\
\hline & 5 & $6.038 \mathrm{e}-01 / 1.781 \mathrm{e}-01$ & $2.254 \mathrm{e}+00 / 3.483 \mathrm{e}-01+$ & $6.122 \mathrm{e}-01 / 5.786 \mathrm{e}-06-$ \\
\hline & 8 & $9.131 \mathrm{e}-01 / 2.210 \mathrm{e}-01$ & $2.545 \mathrm{e}+00 / 3.143 \mathrm{e}-02+$ & $5.263 \mathrm{e}-01 / 1.969 \mathrm{e}-01-$ \\
\hline & 10 & $1.083 e-01 / 3.899 e-02$ & $2.602 \mathrm{e}+00 / 1.752 \mathrm{e}-02+$ & $6.884 \mathrm{e}-01 / 1.474 \mathrm{e}-02+$ \\
\hline \multirow{4}{*}{ DTLZ3 } & 3 & $1.076 \mathrm{e}+00 / 6.160 \mathrm{e}-01$ & $2.210 \mathrm{e}+00 / 1.586 \mathrm{e}+00+$ & $5.469 \mathrm{e}-02 / 2.515 \mathrm{e}-04-$ \\
\hline & 5 & $9.215 \mathrm{e}-01 / 2.894 \mathrm{e}-02$ & $5.051 \mathrm{e}+01 / 1.237 \mathrm{e}+01+$ & 2.126e-01/3.097e-04- \\
\hline & 8 & $9.365 \mathrm{e}-01 / 1.251 \mathrm{e}-02$ & $1.489 \mathrm{e}+03 / 2.775 \mathrm{e}+02+$ & $1.514 \mathrm{e}+00 / 1.589 \mathrm{e}+00+$ \\
\hline & 10 & $9.425 \mathrm{e}-01 / 1.734 \mathrm{e}-01$ & $1.655 \mathrm{e}+03 / 1.664 \mathrm{e}+02+$ & $5.013 \mathrm{e}+00 / 7.685 \mathrm{e}-02+$ \\
\hline \multirow{4}{*}{ DTLZ4 } & 3 & $5.665 \mathrm{e}-01 / 1.727 \mathrm{e}-02$ & $2.595 \mathrm{e}-01 / 1.961 \mathrm{e}-01-$ & $2.980 \mathrm{e}-01 / 3.444 \mathrm{e}-01-$ \\
\hline & 5 & $5.686 \mathrm{e}-01 / 6.550 \mathrm{e}-04$ & $2.491 \mathrm{e}+00 / 1.863 \mathrm{e}-02+$ & $3.214 \mathrm{e}-01 / 1.544 \mathrm{e}-01-$ \\
\hline & 8 & $6.057 \mathrm{e}-01 / 5.477 \mathrm{e}-03$ & $2.596 \mathrm{e}+00 / 2.815 \mathrm{e}-02+$ & $6.156 \mathrm{e}-01 / 4.415 \mathrm{e}-03+$ \\
\hline & 10 & $6.997 \mathrm{e}+00 / 1.970 \mathrm{e}-02$ & $2.636 \mathrm{e}+00 / 7.526 \mathrm{e}-03+$ & $7.006 \mathrm{e}-01 / 1.008 \mathrm{e}-02+$ \\
\hline \multirow{4}{*}{ WFG1 } & 3 & $2.032 \mathrm{e}-01 / 3.044 \mathrm{e}-02$ & 1.161e-01/3.519e-01- & $1.460 \mathrm{e}-01 / 3.706 \mathrm{e}-03-$ \\
\hline & 5 & $2.484 \mathrm{e}-01 / 1.987 \mathrm{e}-01$ & 1.373e-01/8.909e-02- & $4.981 \mathrm{e}-01 / 1.562 \mathrm{e}-02+$ \\
\hline & 8 & $3.132 \mathrm{e}+00 / 2.768 \mathrm{e}-01$ & $2.650 \mathrm{e}+00 / 1.652 \mathrm{e}-01-$ & $1.060 \mathrm{e}+00 / 7.159 \mathrm{e}-02-$ \\
\hline & 10 & $1.048 \mathrm{e}+00 / 9.798 \mathrm{e}-03$ & $2.291 \mathrm{e}+00 / 3.834 \mathrm{e}-03+$ & $1.973 \mathrm{e}+00 / 1.257 \mathrm{e}-01+$ \\
\hline \multirow{4}{*}{ WFG2 } & 3 & $1.353 \mathrm{e}-01 / 4.114 \mathrm{e}-01$ & $3.556 \mathrm{e}-01 / 5.634 \mathrm{e}-02-$ & $1.862 \mathrm{e}-01 / 2.258 \mathrm{e}-03-$ \\
\hline & 5 & $5.442 \mathrm{e}-01 / 1.337 \mathrm{e}+00$ & $1.147 \mathrm{e}-01 / 8.682 \mathrm{e}-02-$ & $7.926 \mathrm{e}-01 / 3.736 \mathrm{e}-02+$ \\
\hline & 8 & $5.253 \mathrm{e}+00 / 1.112 \mathrm{e}+00$ & $2.489 \mathrm{e}+00 / 2.413 \mathrm{e}-01-$ & $4.553 \mathrm{e}+00 / 5.976 \mathrm{e}-01-$ \\
\hline & 10 & $1.136 \mathrm{e}+00 / 3.490 \mathrm{e}+00$ & $3.863 \mathrm{e}+00 / 1.679 \mathrm{e}+00+$ & $5.943 \mathrm{e}+00 / 8.279 \mathrm{e}-02+$ \\
\hline \multirow{4}{*}{ WFG4 } & 3 & $1.504 \mathrm{e}-01 / 2.917 \mathrm{e}-01$ & $3.595 \mathrm{e}-01 / 6.572 \mathrm{e}-03+$ & $2.210 \mathrm{e}-01 / 1.228 \mathrm{e}-04+$ \\
\hline & 5 & $3.559 \mathrm{e}+00 / 2.788 \mathrm{e}-01$ & $1.381 \mathrm{e}+00 / 5.951 \mathrm{e}-02-$ & $1.226 \mathrm{e}+00 / 5.037 \mathrm{e}-04-$ \\
\hline & 8 & $5.699 \mathrm{e}+00 / 6.429 \mathrm{e}-04$ & $3.524 \mathrm{e}+00 / 1.321 \mathrm{e}-01-$ & $3.547 \mathrm{e}+00 / 1.260 \mathrm{e}-02-$ \\
\hline & 10 & $7.940 \mathrm{e}+00 / 3.364 \mathrm{e}-01$ & $5.038 \mathrm{e}+00 / 4.985 \mathrm{e}-02-$ & $6.066 \mathrm{e}+00 / 2.834 \mathrm{e}-01-$ \\
\hline \multirow{4}{*}{ WFG9 } & 3 & $9.152 \mathrm{e}-01 / 3.478 \mathrm{e}-01$ & $4.558 \mathrm{e}-01 / 6.338 \mathrm{e}-02-$ & $2.211 \mathrm{e}-01 / 1.854 \mathrm{e}-04-$ \\
\hline & 5 & $1.296 e+00 / 6.456 e-01$ & $1.691 \mathrm{e}+00 / 2.735 \mathrm{e}-02-$ & $1.213 \mathrm{e}+00 / 1.639 \mathrm{e}-02-$ \\
\hline & 8 & $4.750 \mathrm{e}+00 / 1.342 \mathrm{e}-01$ & $3.754 \mathrm{e}+00 / 3.412 \mathrm{e}-02-$ & $3.570 \mathrm{e}+00 / 1.802 \mathrm{e}-02-$ \\
\hline & 10 & $5.310 \mathrm{e}+00 / 7.865 \mathrm{e}-01$ & $5.513 \mathrm{e}+00 / 5.554 \mathrm{e}-02+$ & $5.681 \mathrm{e}+00 / 2.369 \mathrm{e}-01+$ \\
\hline
\end{tabular}


Table 12 Mean and standard deviation results of IGD obtained by Tk-MaOEA and K-RVEA.

\begin{tabular}{|c|c|c|c|c|c|c|c|c|}
\hline Problem & \multicolumn{4}{|c|}{ DTLZ1 } & \multicolumn{4}{|c|}{ DTLZ2 } \\
\hline M & 3 & 5 & 8 & 10 & 3 & 5 & 8 & 10 \\
\hline \multirow[t]{2}{*}{ Tk-MaOEA } & $1.635 \mathrm{e}-02$ & $1.682 \mathrm{e}-02$ & $1.854 \mathrm{e}-01$ & $1.796 \mathrm{e}-01$ & $6.370 \mathrm{e}-02$ & $1.840 \mathrm{e}-01$ & $2.133 \mathrm{e}-01$ & $1.086 \mathrm{e}-01$ \\
\hline & $/ 9.332 \mathrm{e}-03$ & $/ 7.609 \mathrm{e}-03$ & $/ 1.062 \mathrm{e}-03$ & $/ 2.221 \mathrm{e}-03$ & /3.803e-02 & $/ 1.712 \mathrm{e}-01$ & $/ 2.244 \mathrm{e}-01$ & $/ 3.867 \mathrm{e}-02$ \\
\hline \multirow{2}{*}{ K-RVEA } & $1.7453 \mathrm{e}+01$ & $2.5972 \mathrm{e}+01$ & $2.3388 \mathrm{e}+01$ & $2.0353 \mathrm{e}+01$ & $6.7543 \mathrm{e}-02$ & $2.4301 \mathrm{e}-01$ & $4.2209 \mathrm{e}-01$ & $5.8058 \mathrm{e}-01$ \\
\hline & $/ 8.69 \mathrm{e}+00+$ & $/ 4.11 \mathrm{e}+00+$ & $/ 4.08 \mathrm{e}+00+$ & $/ 9.87 \mathrm{e}+00+$ & $/ 3.08 \mathrm{e}-03+$ & $/ 2.48 \mathrm{e}-02+$ & $/ 1.06 \mathrm{e}-02+$ & $/ 4.17 \mathrm{e}-02+$ \\
\hline Problem & \multicolumn{4}{|c|}{ DTLZ3 } & \multicolumn{4}{|c|}{ DTLZ4 } \\
\hline M & 3 & 5 & 8 & 10 & 3 & 5 & 8 & 10 \\
\hline \multirow[t]{2}{*}{ Tk-MaOEA } & $1.075 e-02$ & $9.216 \mathrm{e}-01$ & $9.365 \mathrm{e}-01$ & $9.425 \mathrm{e}-01$ & $5.656 \mathrm{e}-01$ & $8.686 \mathrm{e}-01$ & $1.056 \mathrm{e}-01$ & $1.197 \mathrm{e}-01$ \\
\hline & /6.121e-01 & $/ 2.885 \mathrm{e}-02$ & $/ 1.242 \mathrm{e}-02$ & $/ 1.755 \mathrm{e}-01$ & $/ 1.711 \mathrm{e}-02$ & /6.566e-04 & $/ 5.472 \mathrm{e}-03$ & $/ 1.950 \mathrm{e}-02$ \\
\hline \multirow{2}{*}{ K-RVEA } & $2.3148 \mathrm{e}+02$ & $2.4395 \mathrm{e}+02$ & $2.3285 \mathrm{e}+02$ & $2.2871 \mathrm{e}+02$ & $9.6446 \mathrm{e}-02$ & $3.5125 \mathrm{e}-01$ & $5.3281 \mathrm{e}-01$ & $6.1738 \mathrm{e}-01$ \\
\hline & $/ 1.72 \mathrm{e}+01+$ & $/ 1.71 \mathrm{e}+01+$ & $/ 3.15 \mathrm{e}+01+$ & $/ 1.29 \mathrm{e}+01+$ & $/ 1.11 \mathrm{e}-02$ & /4.29e-02- & $/ 7.47 \mathrm{e}-02+$ & $/ 4.70 \mathrm{e}-02+$ \\
\hline Problem & \multicolumn{4}{|c|}{ WFG1 } & \multicolumn{4}{|c|}{ WFG4 } \\
\hline M & 3 & 5 & 8 & 10 & 3 & 5 & 8 & 10 \\
\hline \multirow[t]{2}{*}{ Tk-MaOEA } & $1.760 \mathrm{e}-01$ & $5.407 \mathrm{e}-01$ & $1.126 \mathrm{e}-01$ & $2.175 e-01$ & $1.896 \mathrm{e}-01$ & $3.332 \mathrm{e}+00$ & $6.010 \mathrm{e}+00$ & $4.793 e+00$ \\
\hline & /5.230e-03 & /4.966e-02 & $/ 8.790 \mathrm{e}-02$ & /3.111e-02 & $/ 2.134 \mathrm{e}-01$ & $/ 5.442 \mathrm{e}-01$ & $/ 1.690 \mathrm{e}-01$ & $/ 2.844 \mathrm{e}-01$ \\
\hline \multirow{2}{*}{ K-RVEA } & $1.5153 \mathrm{e}+00$ & $2.1045 \mathrm{e}+00$ & $2.8107 \mathrm{e}+00$ & $3.0985 \mathrm{e}+00$ & $3.6579 \mathrm{e}-01$ & $9.4829 \mathrm{e}-01$ & $2.4612 \mathrm{e}+00$ & $3.6256 \mathrm{e}+00$ \\
\hline & $/ 8.22 \mathrm{e}-03+$ & $/ 4.37 \mathrm{e}-03+$ & $/ 1.38 \mathrm{e}-01+$ & /3.32e- $02+$ & $/ 2.62 \mathrm{e}-02+$ & /2.09e-02- & $/ 4.30 \mathrm{e}-02-$ & /2.33e-02- \\
\hline
\end{tabular}

Table 12 shows the comparative results obtained by Tk-MaOEA and K-RVEA on the DTLZ and WFG problems with 3, 5, 8 and 10 objectives. From this table, it can be observed that, TkMaOEA outperforms K-RVEA on most of the test functions. To be specific, TkMaOEA achieves the first rank on DTLZ1, DTLZ2, DTLZ3 and WFG1, respectively. On DTLZ3 and WFG1, TkMaOEA exhibits an obvious performance advantage, obtaining better IGD results with several orders of magnitude than that of K-RVEA. Only on DTLZ4 and WFG4, K-RVEA obtains better results than TkMaOEA. However, TkMaOEA still obtains satisfactory results on high-dimensional DTLZ4 instances, e.g., DTLZ4 with 8, and 10 objectives. Generally, TkMaOEA performs the best on 19 out of the 24 test instances, while K-RVEA does the best only on 5 test instances. 
Table 13 Mean and standard deviation results of HV obtained by Tk-MaOEA, TEMO-MPS and ParEGO

\begin{tabular}{|c|c|c|c|c|c|c|c|}
\hline \multirow{3}{*}{ Problems } & \multirow{3}{*}{ Bound } & \multicolumn{6}{|c|}{500 Generations } \\
\hline & & \multicolumn{2}{|c|}{ ParEGO } & \multicolumn{2}{|c|}{ TEMO-MPS } & \multicolumn{2}{|c|}{ TkMaOEA } \\
\hline & & Mean & Std & Mean & Std & Mean & Std \\
\hline DTLZ1b - 10,1 & 75,75 & 0.6214 & 0.0114 & 0.6822 & 0.0115 & 0.6994 & 0.0010 \\
\hline DTLZ1b - 20,1 & 75,75 & 0.5720 & 0.0128 & 0.6263 & 0.0090 & 0.6391 & 0.0031 \\
\hline DTLZ1b - 30,1 & 75,75 & 0.5201 & 0.0106 & 0.5628 & 0.0090 & 0.5787 & 0.0067 \\
\hline DTLZ1b - 10,2 & 120,120 & 0.4996 & 0.0075 & 0.5291 & 0.0069 & $\mathbf{0 . 5 3 2 7}$ & 0.0043 \\
\hline DTLZ1b - 20,2 & 120,120 & 0.4263 & 0.0074 & 0.4475 & 0.0061 & 0.4458 & 0.0040 \\
\hline DTLZ1b - 30,2 & 120,120 & 0.3528 & 0.0070 & 0.3705 & 0.0064 & 0.3652 & 0.0049 \\
\hline DTLZ1b - 10,3 & 200,200 & 0.5830 & 0.0107 & 0.6070 & 0.0067 & 0.6211 & 0.0040 \\
\hline DTLZ1b - 20,3 & 200,200 & 0.5206 & 0.0075 & 0.5397 & 0.0067 & 0.5509 & 0.0031 \\
\hline DTLZ1b - 30,3 & 200,200 & 0.4509 & 0.0071 & 0.4695 & 0.0070 & 0.4765 & 0.0053 \\
\hline DTLZ3b - 10,1 & $150,150,150$ & 0.5187 & 0.0320 & 0.5825 & 0.0307 & 0.6974 & 0.0032 \\
\hline DTLZ3b - 20,1 & $150,150,150$ & 0.4174 & 0.0350 & 0.4868 & 0.0304 & 0.6049 & 0.0094 \\
\hline DTLZ3b - 30,1 & $150,150,150$ & 0.3204 & 0.0201 & 0.3748 & 0.0249 & 0.5008 & 0.0060 \\
\hline DTLZ3b - 10,2 & $300,300,300$ & 0.5562 & 0.0362 & 0.6016 & 0.0274 & 0.7064 & 0.0045 \\
\hline DTLZ3b - 20,2 & $300,300,300$ & 0.4613 & 0.0241 & 0.4949 & 0.0269 & 0.6155 & 0.0094 \\
\hline DTLZ3b - 30,2 & $300,300,300$ & 0.3420 & 0.0249 & 0.3590 & 0.0196 & 0.5133 & 0.0047 \\
\hline DTLZ3b - 10,3 & $450,450,450$ & 0.5871 & 0.0325 & 0.5667 & 0.0353 & 0.7075 & 0.0032 \\
\hline DTLZ3b - 20,3 & $450,450,450$ & 0.4694 & 0.0290 & 0.4524 & 0.0249 & 0.6181 & 0.0084 \\
\hline DTLZ3b - 30,3 & $450,450,450$ & 0.3636 & 0.0261 & 0.3596 & 0.0203 & 0.5097 & 0.0083 \\
\hline
\end{tabular}

\subsubsection{Comparison results with transfer-learning-based algorithms}

In order to further investigate the algorithm's effectiveness, we employ two transfer-learning-based algorithms TEMO-MPS [75] and ParEGO [76] to compared with Tk-MaOEA. TEMO-MPS [75], an improved ParEGO algorithm, exploits multi-problem surrogates to achieve knowledge transfer for MOPs. In principle, TEMO-MPS is an adaptive knowledge-reuse-based MOEA. ParEGO [76] aims to utilize the probabilistic nature of Gaussian process regression models to enhance the search in multi-objective objective space. More details can refer to [76]. In this experiment, the parameter setting of Tk-MaOEA follows that of Section 4.2.

The statistical results in terms of mean and standard deviation of HV values obtained by Tk-MaOEA, TEMO-MPS and ParEGO, are given in Table 13. Note that, reference [75] has reported the statistical HV results of TEMO-MPS and ParEGO on the DTLZ1 and DTLZ3 variants, thereby these results are directly listed in Table 13. The detailed formulations regarding the DTLZ1 and DTLZ3 variants, as shown in [75], have two major modifications: a) the number of local fronts is reduced, and the value $20 \pi$ within the cosine term of the original 
DTLZ1 is replaced by $2 \pi$, and b) the function is combined with two variables to create a suite of related optimization tasks. The two test function sets are respectively denoted by DTLZ1b- $\delta 1, \delta 2$ or DTLZ3b- $\delta 1, \delta 2$. It is clear that the larger difference between $\delta 1$ and $\delta 2$ means the lower similarity. In this experiment, $\delta 1 \in\{10,20,30\}$ and $\delta 2 \in\{1,2,3\}$ are set to generate 18 synthetic multimodal DTLZ functions, as shown in Table 13.

From Table 13, it is observed that TkMaOEA shows a performance superiority to other algorithms. For DTLZ1b, TEMO-MPS obtains the first ranks on DTLZ1b-20, 2 and DTLZ1b-30, 2. In fact, the statistical results in terms of HV values of Tk-MaOEA are very close to that of TEMO-MPS on DTLZ1b-20, 2 and DTLZ1b-30, 2. For other test functions, e.g., DTLZ1b-10, 1-3, DTLZ1b-20, 1, 3, DTLZ1b-30, 1, 3, and all DTLZ3b instances, Tk-MaOEA obtains the first ranks, performing better than TEMO-MPS. This may be due to the fact that TEMO-MPS aggregates all objective functions into a single objective function, which causes the inaccurate identification of new candidate solutions during the search. For DTLZ3b, TkMaOEA outperforms ParEGO and TEMO-MPS on all the test instances. This can be explained that Tk-MaOEA can accelerate the convergence of solutions by using the transfer matrix, while TEMO-MPS only aggregates the objective values only through a random vector, resulting in an inaccurate representation for the corresponding solution.

\section{Conclusions}

In order to alleviate the effect of the curse of dimensionality in MaOPs, this paper develops a novel evolutionary optimization framework, called Tk-MaOEA, based on transfer learning assisted by Kriging model. The aim of Tk-MaOEA is to enhance the selection pressure in fitness evaluation in MaOPs. At the global space optimization level, transfer learning is used to reduce the number of redundant objectives, by the means of the deliberately designed transfer matrix. At the objective optimization level, Kriging model is incorporated for each objective to further reduce the optimization complexity during the evolutionary process. In addition, the fast non-dominated sorting and FCS strategies are incorporated in environmental selection to save and retrieve the final non-dominated solutions.

The proposed Tk-MaOEA has been experimentally compared with several popular MaOEAs including NSGA-III, MOEA/D, MOMBII and VaEA on a set of well-defined test benchmarks. Experimental results show that Tk-MaOEA is significant superior or at least comparable to its compared algorithms in terms of two commonly used metrics IGD and HV. It should be noted that Tk-MaOEA sometimes encounters the dilemma of being easily trapped into local many-objective optima on some test problems Accordingly, we do not declare that 
Tk-MaOEA is always superior to other MaOEAs. The strengths and weaknesses of Tk-MaOEA need to be investigated further, especially according to the specific features of the problems. A comprehensive sensitivity analysis of the algorithm's parameters, and the research on real-world applications will be highlighted in our future work.

\section{APPENDIX}

The process of transfer learning that aims to reduce the number of objectives, is based on an important premise that the properties of original objectives should be maintained as much as possible. Thus, the following definitions are given and the proposed theorems with respects to the transfer matrix are proven mathematically.

Definition 1: for a population matrix $P$, the transferred matrix $\operatorname{Tr}$ can be transformed from $P$ based on the best individual in $\mathrm{P}$, by using a transfer matrix $\mathrm{T}$, then

$$
\operatorname{Tr}=P * T
$$

Theorem 1: In Definition 1, the size of matrix $\mathrm{T}_{\mathrm{r}}$ should be $\left(N^{*} T N\right)$, where $N$ is the number of individuals in population $P$, and $T N$ is the number of objectives that has been reduced.

Proof: Considering that the size of matrix $P$ is $\left(N^{*} M\right)$, the size of matrix $T$ should be $\left(M^{*} T N\right)$. According to the matrix multiplication in Definition 1, we can get that $\operatorname{Tr}=\mathrm{P}_{\mathrm{N}^{*} \mathrm{M}}{ }^{*} \mathrm{~T}_{\mathrm{M}}{ }^{*} \mathrm{TN}$, and the size of $\operatorname{Tr}$ is $\left(N^{*} T N\right)$. Therefore, Theorem 1 is proven.

Definition 2: Given an individual $p_{t}^{m}$ in population $\mathrm{P}_{\mathrm{t}}, \forall p_{t}^{m}=\left(x_{t_{1}}^{m}, x_{t_{2}}^{m}, \cdots, x_{t_{M}}^{m}\right), m=1,2, \ldots N$, for a column vector in the transfer matrix, $\forall t_{j}=\left(t_{1 j}, t_{2 j}, \cdots, t_{M j}\right)^{T}, j=1,2, \ldots N$, and an individual $t r_{i}^{j}$ in the population $\operatorname{Tr}$,

$$
\begin{aligned}
& \forall t r_{t}^{j}=\left(y_{t_{1}}^{j}, y_{t_{2}}^{j}, \cdots, y_{t_{T N}}^{j}\right), j=1,2, \ldots N, \text { according to Definition } \mathbf{1} \text {, there exists a relation between them as } \\
& \qquad \begin{aligned}
y_{k}^{m} & =p_{t}^{m} * t_{k}, \quad m=1,2, \cdots N \text { and } k=1,2, \cdots T N \\
& =\sum_{i=1}^{M} x_{i}^{m} * t_{i}^{j}=<p_{t}^{m}, t_{k}>
\end{aligned}
\end{aligned}
$$

Theorem 2: for an individual $t r_{i}^{j}$ in the population $\operatorname{Tr} \forall t r_{t}^{j}=\left(y_{t_{1}}^{j}, y_{t_{2}}^{j}, \cdots, y_{t_{T N}}^{j}\right), j=1,2, \ldots N$, each set of coordinates is the map length in direction of the each column vector in transfer matrix .

Proof: In the implementation of matrix multiplication, the row of previous matrix multiplies the column of the matrix, as depicted in Definition 2. According to the formula related to the inner product (i.e., Eq.(12)) and the function $\cos$ related to the angle between two vectors (i.e., Eq.(13)), if the length of $t_{k}$ is 1 , the formula of inner product (i.e., Eq.(14)) is the map length in the direction of the $t_{\text {k.. }}$ Therefore, the Theorem 2 is proven. 


$$
\begin{gathered}
<p_{t}^{m}, t_{k}>=\sum_{i=1}^{M} x_{i}^{m} * t_{i}^{j} \\
\cos \left(p_{t}^{m}, t_{k}\right)=\frac{\sum_{i=1}^{M} x_{i}^{m} * t_{i}^{j}}{\left\|p_{t}^{m}\right\| *\left\|t_{k}\right\|} \\
<p_{t}^{m}, t_{k}>=\sum_{i=1}^{M} x_{i}^{m} * t_{i}^{j}=\left\|p_{t}^{m}\right\| * \cos \left(p_{t}^{m}, t_{k}\right)
\end{gathered}
$$

\section{Acknowledgements}

This work is supported by National Natural Science Foundation of China under Grant No. 6177021519, 61503373, and 61572123; National Science Foundation for Distinguished Young Scholars of China under Grant No. 71325002 .

\section{References}

[1] E. Zitzler and L. Thiele, "Multiobjective evolutionary algorithms: A comparative case study and the strength Pareto approach,” IEEE Trans. Evol. Comput., vol. 3, no. 4, pp. 257-271, Nov. 1999.

[2] R. Wang, S. Lai, G. Wu, L.Xing, L. Wang, H. Ishibuchide, "Multi-clustering via evolutionary multi-objective optimization," Information Sciences, 2018, 450,pp.128-140.

[3] Y. Yuan, H. Xu, B. Wang, et al. "Balancing Convergence and Diversity in Decomposition-Based Many-Objective Optimizers IEEE Trans. Evol. Comput., vol. 20, no. 2, pp.180-198, 2015

[4] J. G. Herrero, A. Berlanga, and J. M. M. Lopez, "Effective evolutionary algorithms for many-specifications attainment: Application to air traffic control tracking filters,” IEEE Trans. Evol. Comput., vol. 13, no. 1, pp. 151-168, Feb. 2009.

[5] L. Ma, S.Cheng, X. Wang, M.Huang, H. Shen, X. He, Y. Shi, "Cooperative Two-engine Multi-objective Bee Foraging Algorithm with Reinforcement Learning," Knowledge-Based Systems,133(2017):278-293,2017.

[6] N. Chen, W. Chen, Y. Gong et al. An Evolutionary Algorithm with Double-Level Archives for Multiobjective Optimization., IEEE Transactions on Cybernetics, 2015, 45(9):1851.

[7] Y. Xiang, Y. Zhou, M. Li, et al. "A Vector Angle-Based Evolutionary Algorithm for Unconstrained Many-Objective Optimization", IEEE Transactions on Evolutionary Computation, 2017, 21(1):131-152.

[8] L. Ma, K. Hu, Y. Zhu, H. Chen. Cooperative Artificial Bee Colony Algorithm for Multi-objective RFID Network Planning, Journal of network and computer applications, 42(2014)143-162.

[9] V. Khare, X. Yao, and K. Deb, "Performance scaling of multi-objective evolutionary algorithms," in Proc. Evol. Multi-Criterion Optim., Faro, Portugal, 2003, pp. 376-390.

[10] K. Deb, A. Pratap, S. Agarwal, and T. Meyarivan, A fast and elitist multiobjective genetic algorithm: NSGA-II, IEEE Trans. Evol. Comput., vol. 6, no. 2, pp. 182-197, Apr. 2002.

[11] E. Zitzler, M. Laumanns, and L. Thiele, SPEA2: Improving the strength pareto evolutionary algorithm, in Proc. EUROGEN 2001: Evolutionary Methods Design Optimization Control Appl. Ind. Problems, Athens, Greece, 2002, pp. 
$95-100$.

[12] H. Ishibuchi, N. Tsukamoto, and Y. Nojima, "Evolutionary manyobjective optimization: A short review," in Proc. IEEE Congr. Evol. Comput., Jun. 2008, pp. 2424-2431.

[13] M. K"oppen and K. Yoshida, "Substitute distance assignments in NSGAII for handling many-objective optimization problems," in Proc. EMO, vol. 4403. 2007, pp. 727-741.

[14] Z. He and G. G. Yen, "Fuzzy-based Pareto optimality for many-objective evolutionary algorithms," IEEE Trans. Evol. Comput., vol. 18, no. 2, pp. 269-285, Apr. 2014.

[15] Z. He, G. G. Yen. Many-Objective Evolutionary Algorithm: Objective Space Reduction and Diversity Improvement[J]. IEEE Transactions on Evolutionary Computation, 2016, 20(1):145-160.

[16] H. Sato, H. Aguirre, and K. Tanaka, "Variable space diversity, crossover and mutation in MOEA solving many-objective knapsack problems," Ann. Math. Artif. Intell., vol. 68, no. 4, pp. 197-224, 2013.

[17] H. Ishibuchi, Y. Tanigaki, H. Masuda, and Y. Nojima, "Distance-based analysis of crossover operators for many-objective knapsack problems," in Proc. Int. Conf. Parallel Prob. Solv. Nat., Ljubljana, Slovenia, 2014, pp. $600-610$.

[18] K. Deb and D. Saxena, "On finding Pareto-optimal solutions through dimensionality reduction for certain large-dimensional multi-objective optimization problems," Kanpur Genet. Algorithms Lab., Indian Inst. Technol. Kanpur, Kanpur, India, KanGAL Tech. Rep. 2005011, 2005.

[19] H. Singh, A. Isaacs, and T. Ray, "A Pareto corner search evolutionary algorithm and dimensionality reduction in many-objective optimization problems,” IEEE Trans. Evol. Comput., vol. 15, no. 4, pp. 539-556, 2011.

[20] Q. Zhang and H. Li, “MOEA/D: A multiobjective evolutionary algorithm based on decomposition,” IEEE Trans. Evol. Comput., vol. 11, no. 6, pp. 712-731, Dec. 2007.

[21] N. Chen, W. N. Chen, Y. J. Gong, et al. An Evolutionary Algorithm with Double-Level Archives for Multiobjective Optimization, IEEE Transactions on Cybernetics, 2015, 45(9):1851-1863.

[22] Q. Zhang, W. Liu, E. Tsang, and B. Virginas, "Expensive multiobjective optimization by MOEA/D with Gaussian process model,” IEEE Trans. Evol. Comput., vol. 14, no. 3, pp. 456-474, Jun. 2010.

[23] E. Zitzler and S. Künzli, "Indicator-based selection in multiobjective search," in Parallel Problem Solving from Nature-PPSN VIII. Berlin, Germany: Springer, 2004, pp. 832-842.

[24] N. Beume, B. Naujoks, M. Emmerich, SMS-EMOA: Multiobjective selection based on dominated hypervolume. European Journal of Operational Research, 2007, 181(3):1653-1669.

[25] Hernandez Gomez R, CoelloCoello C. MOMBI: A new metaheuristic for many-objective optimization based on the R2 indicator. Evolutionary Computation, 2013:2488-2495.

[26] J. Bader, E. Zitzler, HypE: an algorithm for fast hypervolume-based many-objective optimization. Evolutionary Computation, 2011, 19(1):45-76.

[27] K. Deb, M. Mohan, S. Mishra, Evaluating the $\varepsilon$-domination based multi-objective evolutionary algorithm for a quick computation of Pareto-optimal solutions, Evol. Comput., vol. 13, no. 4, pp. 501-525, 2005.

[28] K. Ikeda, H. Kita, S. Kobayashi, Failure of Pareto-based MOEAs: does non-dominated really mean near to optimal. Evolutionary Computation, 2001. Proceedings of the 2001 Congress on. IEEE, 2001:957-962 vol. 2.

[29] H. Sato, H. E. Aguirre, K. Tanaka, Controlling dominance area of solutions and its impact on the performance of 
MOEAs, in Evolutionary Multi-Criterion Optimization. Berlin, Germany: Springer, 2007, pp. 5-20.

[30] Z. Xiufen, C. Yu, L. Minzhong, et al., A new evolutionary algorithm for solving many-objective optimization problems. Systems Man \& Cybernetics Part B Cybernetics IEEE Transactions on, 2008, 38(5):1402-12.

[31] S. Kukkonen, J. Lampinen, "Ranking-dominance and many-objective optimization," in Proc. IEEE Congr. Evol. Comput. (CEC), Singapore, 2007, pp. 3983-3990.

[32] X. Zou, Y. Chen, M. Liu, and L. Kang, "A new evolutionary algorithm for solving many-objective optimization problems,” IEEE Trans. Syst., Man, Cybern. B, Cybern., vol. 38, no. 5, pp. 1402-1412, Oct. 2008.

[33] S. Yang,, M. Li, X. Liu, J. Zheng, A grid-based evolutionary algorithm for many-objective optimization. Evolutionary Computation, IEEE Transactions on, 2013. 17(5), pp. 721--736.

[34] S. Kukkonen, J. Lampinen, "Ranking-dominance and many-objective optimization," in Proc. IEEE Congr. Evol. Comput. (CEC), Singapore, 2007, pp. 3983-3990.

[35] K. Deb, H. Jain, “An evolutionary many-objective optimization algorithm using reference-point-based nondominated sorting approach, part I: Solving problems with box constraints,” IEEE Trans. Evol. Comput., vol. 18, no. 4, pp. 577-601, Aug. 2014.

[36] H. Wang, L. Jiao, X. Yao, Two_Arch2: An Improved Two-Archive Algorithm for Many-Objective Optimization. IEEE Transactions on Evolutionary Computation, 2015, 19(4):524-541.

[37] R. Cheng, Y. Jin, Olhofer M, et al. A Reference Vector Guided Evolutionary Algorithm for Many-Objective Optimization. IEEE Transactions on Evolutionary Computation, 2016.

[38] H. K. Singh, A. Isaacs, T. Ray, A Pareto Corner Search Evolutionary Algorithm and Dimensionality Reduction in Many-Objective Optimization Problems. Evolutionary Computation IEEE Transactions on, 2011, 15(4):539-556.

[39] S. Bandyopadhyay, A. Mukherjee, An Algorithm for Many-Objective Optimization With Reduced Objective Computations: A Study in Differential Evolution. IEEE Transactions on Evolutionary Computation, 2015, 19(3):400-413.

[40] L. Ma, S.Cheng, X. Wang, M.Huang, H. Shen, X. He, Y. Shi. Cooperative Two-engine Multi-objective Bee Foraging Algorithm with Reinforcement Learning, Knowledge-Based Systems,133(2017):278-293,2017.

[41] D. Brockhoff and E. Zitzler, "Are all objectives necessary? On dimensionality reduction in evolutionary multiobjective optimization,” in Proc. 9th PPSN, LNCS 4193. 2006, pp. 533-542.

[42] D. Brockhoff and E. Zitzler, "Objective reduction in evolutionary multiobjective optimization: Theory and applications,” Evol. Comput., vol. 17, no. 2, pp. 135-166, 2009.

[43] A. L. Jaimes, C. A. C. Coello, and D. Chakraborty, "Objective reduction using a feature selection technique," in Proc. GECCO, 2008, pp. 673-680.

[44] H. K. Singh, A. Isaacs, and T. Ray, "A Pareto corner search evolutionary algorithm and dimensionality reduction in many-objective optimization problems,” IEEE Trans. Evol. Comput., vol. 99, no. 4, pp. 1-18, Aug. 2011.

[45] K. Deb and D. K. Saxena, "Searching for Pareto-optimal solutions through dimensionality reduction for certain large-dimensional multiobjective optimization problems,” in Proc. IEEE CEC, Jul. 2006, pp.3353-3360.

[46] D. Saxena and K. Deb, "Non-linear dimensionality reduction procedures for certain large-dimensional multiobjective optimization problems: Employing correntropy and a novel maximum variance unfolding," in Evolutionary Multi-Criterion Optimization (Lecture Notes in Computer Science, vol. 4403), S. Obayashi, K. Deb, C. Poloni, T. 
Hiroyasu, and T. Murata, Eds. Berlin/Heidelberg, Germany: Springer, 2007, pp. 772-787.

[47] B. Scholkopf, A. Smola, and K. R. Muller, "Nonlinear component analysis as a kernel eigenvalue problem," Neural Comput., vol. 10, no. 5, pp. 1299-1319, 1998.

[48] L. K. Saul, K. Q. Weinberger, J. H. Ham, F. Sha, and D. D. Lee, "Spectral methods for dimensionality reduction," in Semisupervised Learning, O. C. B. Schoelkopf and A. Zien, Eds. Cambridge, MA: MIT Press, 2006.

[49] K. Q. Weinberger and L. K. Saul, “Unsupervised learning of image manifolds by semidefinite programming,” Int. J. Comput. Vis., vol. 70, no. 1, pp. 77-90, 2006.

[50] A.I.J.Forrester and A. J. Keane, "Recent advances in surrogate-based optimization,” Prog. Aerosp. Sci., vol. 45, no. 1, pp. 50-79, Jan., 2009.

[51] B. Liu, Q. Zhang, and G. G. E. Gielen, "A gaussian process surrogate model assisted evolutionary algorithm for medium scale expensive optimization problems," IEEE Trans. Evol. Comput., vol. 18, no. 2, pp. 180-192, Apr. 2014.

[52] S. Das, S. S. Mullick, and P. N. Suganthan, "Recent advances in differential evolution - An updated survey," Swarm Evol. Comput., vol. 27, pp. 1-30, Apr. 2016.

[53] P. S. Palar, T. Tsuchiya, and G. T. Parks, "A comparative study of local search within a surrogate-assisted multi-objective memetic algorithm framework for expensive problems," Appl. Soft Comput., vol. 43, pp. 1-19, Jun. 2016.

[54] S. C. Horng, S. Y. Lin, "Evolutionary algorithm assisted by surrogate model in the framework of ordinal optimization and optimal computing budget allocation", Information Sciences, 2013, 233(2):214-229.

[55] S. Huband, P. Hingston, L. Barone, and L. While, "A review of multiobjective test problems and a scalable test problem toolkit,” IEEE Trans. Evol. Comput., vol. 10, no. 5, pp. 477-506, Oct. 2006.

[56] H. Yu, Y. Tan, J. Zeng, et al. Surrogate-assisted Hierarchical Particle Swarm Optimization, Information Sciences, 2018, 454-455.

[57] G. Venturelli, E. Benini, and Ł. Łaniewski-Wołłk, “A Kriging-assisted multiobjective evolutionary algorithm,” Appl. Soft Comput., vol. 58, pp. 155-175, Sep. 2017.

[58] H.-J. Park, H.-K. Yeo, S.-Y. Jung, T.-K. Chung, J.-S. Ro, and H.-K. Jung, “A robust multimodal optimization algorithm based on a sub-division surrogate model and an improved sampling method,” IEEE Trans. Magn., vol. 54, no. 3, Mar. 2018, Art. ID 8201704.

[59] Y. Jin, "Surrogate-assisted evolutionary computation: Recent advances and future challenges," Swarm Evol. Comput., vol. 1, no. 2, pp. 61-70, Jun. 2011.

[60] C. Sun, Y. Jin, R. Cheng, J. Ding, and J. Zeng, "Surrogate-assisted cooperative swarm optimization of high-dimensional expensive problems,” IEEE Trans. Evol. Comput., vol. 21, no. 4, pp. 644-660, Aug. 2017.

[61] K. Deb and H. Jain, "An evolutionary many-objective optimization algorithm using reference-point-based nondominated sorting approach, part I: Solving problems with box constraints," IEEE Trans. Evol. Comput., vol. 18, no. 4, pp. 577-601, Aug. 2014.

[62] R. Cheng, Y. Jin, M. Olhofer, et al. A Reference Vector Guided Evolutionary Algorithm for Many-Objective Optimization, IEEE Transactions on Evolutionary Computation, 2016, 20(5):773-791.

[63] B. Chen, W. Zeng, Y. Lin, et al., A New Local Search-Based Multiobjective Optimization Algorithm, IEEE Transactions on Evolutionary Computation, 2014, 19(1):1-1. 
[64] S. Jeong, Y. Minemura, and S. Obayashi, "Optimization of combustion chamber for diesel engine using Kriging model,” J. Fluid Sci. Technol., vol. 1, no. 2, pp. 138-146, Dec. 2006.

[65] R. H. Gómez and C. A. C. Coello, "Improved metaheuristic based on the R2 indicator for many-objective optimization,” in Proc. Genet. Evol. Comput. Conf., Madrid, Spain, 2015, pp. 679-686.

[66] C. A. C. Coello, G. B. Lamont, and D. A. V. Veldhuizen, Evolutionary Algorithms for Solving Multi-Objective Problems, 2nd ed. New York, NY, USA: Springer, 2007.

[67] H. Ishibuchi, H. Masuda, Y. Tanigaki, and Y. Nojima, "Difficulties in specifying reference points to calculate the inverted generational distance for many-objective optimization problems,” in Proc. IEEE Symp. Comput. Intell. Multi-Criteria Decis. Making (MCDM), Orlando, FL, USA, 2014, pp. 170-177.

[68] X. Zhang, Y. Tian, Y. Jin. A Knee Point-Driven Evolutionary Algorithm for Many-Objective Optimization. IEEE Transactions on Evolutionary Computation, 2015, 19(6):761-776.

[69] W. J. Connover, Practical Nonparametric Statistics, 3rd ed. New York, NY, USA: Wiley, 1999, ch. 5.

[70] K. Deb, L. Thiele, M. Laumanns, and E. Zitzler, "Scalable test problems for evolutionary multi-objective optimization," Dept. Comput. Eng. Netw. Lab., ETH Zurich, Zurich, Switzerland, TIK-Tech. Rep. 112, 2001.

[71] Friedman M. A Comparison of Alternative Tests of Significance for the Problem of m Rankings, Annals of Mathematical Statistics, 1940, 11(1):86-92.

[72] A. Toffolo, E. Benini, Genetic diversity as an objective in multi-objectiveevolutionary algorithms, Evol. Comput. 11 (2) (2003) 151-157.

[73] G. Venturelli, E. Benini, Ł. Łaniewski-Wołłk, A Kriging-assisted Multiobjective Evolutionary Algorithm, Applied Soft Computing, 58(2017): 155-175.

[74] Chugh T, Jin Y, Miettinen K, et al. A Surrogate-Assisted Reference Vector Guided Evolutionary Algorithm for Computationally Expensive Many-Objective Optimization[J]. IEEE Transactions on Evolutionary Computation, 2018, 22(1):129-142.

[75] Min A T W, Ong Y S, Gupta A, et al. Multi-Problem Surrogates: Transfer Evolutionary Multiobjective Optimization of Computationally Expensive Problems[J]. IEEE Transactions on Evolutionary Computation, 2017, PP(99):1-1.

[76] Knowles J. ParEGO: a hybrid algorithm with on-line landscape approximation for expensive multiobjective optimization problems[J]. IEEE Transactions on Evolutionary Computation, 2006, 10(1):50-66. 